ARTICLE

\title{
A conserved immunogenic and vulnerable site on the coronavirus spike protein delineated by cross-reactive monoclonal antibodies
}

\author{
Chunyan Wang ${ }^{1}$, Rien van Haperen ${ }^{2,3}$, Javier Gutiérrez-Álvarez (10 ${ }^{4}$, Wentao Li (i) ${ }^{1}$, Nisreen M. A. Okba(i) ${ }^{5}$, \\ Irina Albulescu', Ivy Widjaja,7, Brenda van Dieren', Raul Fernandez-Delgado (1) 4, Isabel Sola (1) ${ }^{4}$, \\ Daniel L. Hurdiss (10) ${ }^{1}$, Olalekan Daramola ${ }^{6}$, Frank Grosveld ${ }^{2,3}$, Frank J. M. van Kuppeveld (1) 1, \\ Bart L. Haagmans (D) ${ }^{5}$, Luis Enjuanes ${ }^{4}$, Dubravka Drabek (iD ${ }^{2,3} \&$ Berend-Jan Bosch (i) ${ }^{1 \times}$
}

The coronavirus spike glycoprotein, located on the virion surface, is the key mediator of cell entry and the focus for development of protective antibodies and vaccines. Structural studies show exposed sites on the spike trimer that might be targeted by antibodies with crossspecies specificity. Here we isolated two human monoclonal antibodies from immunized humanized mice that display a remarkable cross-reactivity against distinct spike proteins of betacoronaviruses including SARS-CoV, SARS-CoV-2, MERS-CoV and the endemic human coronavirus HCoV-OC43. Both cross-reactive antibodies target the stem helix in the spike S2 fusion subunit which, in the prefusion conformation of trimeric spike, forms a surface exposed membrane-proximal helical bundle. Both antibodies block MERS-CoV infection in cells and provide protection to mice from lethal MERS-CoV challenge in prophylactic and/or therapeutic models. Our work highlights an immunogenic and vulnerable site on the betacoronavirus spike protein enabling elicitation of antibodies with unusual binding breadth.

\footnotetext{
${ }^{1}$ Division of Infectious Diseases and Immunology, Department of Biomolecular Health Sciences, Faculty of Veterinary Medicine, Utrecht University, Utrecht, the Netherlands. ${ }^{2}$ Department of Cell Biology, Erasmus Medical Center, Rotterdam, the Netherlands. ${ }^{3}$ Harbour BioMed, Rotterdam, the Netherlands. ${ }^{4}$ Department of Molecular and Cell Biology, National Center for Biotechnology-Spanish National Research Council (CNB-CSIC), Madrid, Spain. ${ }^{5}$ Department of Viroscience, Erasmus Medical Center, Rotterdam, the Netherlands. ${ }^{6}$ Cell Culture and Fermentation Sciences, Biopharmaceutical Development, BioPharmaceuticals R\&D, AstraZeneca, Cambridge, UK. ${ }^{7}$ Present address: Merus N.V., Utrecht, the Netherlands. ${ }^{凶}$ email: b.j.bosch@uu.nl
} 
T he coronavirus disease 2019 (COVID-19) pandemic is caused by the severe acute respiratory syndrome coronavirus 2 (SARS-CoV-2) which emerged in China, in late $2019^{1}$. The virus belongs to the subgenus Sarbecovirus of the genus Betacoronavirus within the subfamily Orthocoronavirinae ${ }^{2}$. Two other zoonotic coronaviruses emerged in the last 20 years and cause severe acute respiratory disease in humans, similar to that seen with SARS-CoV-2. SARS-CoV, which also belongs to the subgenus Sarbecovirus and is closely related to SARS-CoV-2, crossed species barriers to humans in China in 2002, and caused worldwide $\sim 8000$ cases with a $10 \%$ mortality rate before it was contained in $2003^{3}$. Ten years later in 2012, the Middle East respiratory syndrome (MERS) coronavirus (subgenus Merbecovirus, genus Betacoronavirus) surfaced in Saudi Arabia. This virus is recurrently introduced in the human population from a dromedary camel reservoir with limited human-to-human spread and has led to $\sim 2500$ cases with $\sim 35 \%$ of reported patients succumbing to the infection ${ }^{4}$. In addition, four endemic human coronaviruses (HCoVs) circulate in humans, including HCoV-229E and HCoV-NL63 (genus Alphacoronavirus) and HCoV-OC43 and HCoV-HKU1 (subgenus Embecovirus, genus Betacoronavirus). While these viruses are typically associated with mild respiratory illnesses (common colds) $)^{5-7}$, they can cause significant morbitity, and even mortality, in immunocompromised individuals ${ }^{8}$. All these viruses made their way into humans from an animal reservoir, illustrating the zoonotic threat posed by coronaviruses and their pandemic potential ${ }^{1,9-11}$. The devastating socio-economic consequences of the COVID-19 pandemic urges the development of intervention strategies that can mitigate the outbreak of future emerging coronaviruses.

Identification of antibodies that broadly react with existing (human) coronaviruses could be of relevance to increase our level of pandemic preparedness. Such antibodies might be useful for virus diagnostics in the early stages of a pandemic. Moreover, their epitopes may enable the development of antibody-based therapeutics or vaccines that provide broad protection not only against contemporary pathogens but also against those that likely emerge in the future ${ }^{12}$. Although being identified for other virus families ${ }^{13-18}$, exploration of broadly reactive antibodies against coronaviruses is still in its infancy. Analysis of human polyclonal sera indicated that such cross-reactive antibodies might exist as one study reported that $25 \%$ of the convalescent SARS patient sera had low titers of antibodies that could neutralize MERS$\mathrm{CoV}^{19}$. In addition, Barnes et al. ${ }^{20}$ reported reactivity of purified plasma IgG from ten convalescent COVID-19 patients with the MERS-CoV spike protein, and a study by Wec et al. ${ }^{21}$ demonstrated that a subset of SARS-CoV-2 cross-reactive antibodies isolated from a convalescent SARS-CoV donor could react with one or more of the endemic $\mathrm{HCoV}$ spike proteins. However, little, if anything, is known about the functionalities and epitopes of these cross-reactive antibodies.

Coronavirus neutralizing antibodies target the trimeric spike (S) glycoproteins on the viral surface that mediate virus attachment and entry into the host cell. Several cryo-electron microscopy (cryo-EM) structures of trimeric spikes have been determined, with each comprising three S1 receptor-binding subunits that collectively cap the trimeric S2 fusion subunit ${ }^{22-32}$. Most antibodies neutralize coronavirus infection by binding to the receptor-binding subdomain of S1 and blocking receptor interactions. These antibodies are highly species specific and often strain specific due to a high sequence diversity in the receptorbinding sites among coronaviruses, even for those that engage the same receptor (e.g. SARS-CoV and SARS-CoV-2) ${ }^{33}$. Recently S1-targeting monoclonal antibodies have been identified that bind regions distal to the receptor-binding site and that crossneutralize coronaviruses within the Sarbecovirus subgenus ${ }^{21,34-40}$ with in vivo protective efficacy ${ }^{40,41}$, providing potential new leads for the development of a pan-sarbecovirus vaccine. Neutralizing antibodies that broadly target coronaviruses from distinct (sub) genera are less likely to be found as the overall sequence identity among all coronaviruses spike proteins is low. Relative to the S1 subunit, the membrane-anchored S2 subunit, which mediates fusion of the viral and cellular membrane through receptorinduced conformational rearrangements, exhibits a higher level of protein sequence conservation across coronavirus spike proteins. Structural studies show exposed sites on the S2 base of the spike trimer that might be targeted by antibodies with cross-species specificity ${ }^{28,42}$.

Here we report first evidence of a class of S2-targeting antibodies with broad reactivity towards several human betacoronaviruses from distinct subgenera, and characterized their antiviral activity, epitope and in vivo protective efficacy.

\section{Results}

Cross-reactivity of human monoclonal antibodies to betacoronavirus spike proteins. Five out of seven coronaviruses that are currently known to cause disease in humans belong to the Betacoronavirus genus, including SARS-CoV and SARS-CoV-2 (subgenus Sarbecovirus), MERS-CoV (subgenus Merbecovirus), and the endemic HCoVs HCoV-OC43 and HCoV-HKU1 (subgenus Embecovirus). To elicit and isolate betacoronavirus spiketargeting antibodies with cross-species specificity, we earlier immunized mice with trimeric spike ectodomains $\left(\mathrm{S}_{\text {ecto }}\right)$ of three human-infecting betacoronaviruses from different subgenera (HCoV-OC43, SARS-CoV and MERS-CoV) following a sequential immunization scheme as described in Supplementary Fig. $1 \mathrm{a}^{34}$. Transgenic H2L2 mice that encode the human immunoglobulin repertoire were used for immunization to develop fully human antibodies. We selected 203 hybridomas based on reactivity against at least one of the three spike antigens and screened their supernatants for cross-reactivity against five distinct betacoronavirus spike proteins by ELISA (Supplementary Fig. 1b). Based on their broad ELISA reactivity profile towards multiple coronavirus spike proteins, we selected antibody 28D9 as well as a previously isolated MERS-S human monoclonal antibody $1.6 \mathrm{C}^{43}$. Antibodies $1.6 \mathrm{C} 7$ and $28 \mathrm{D} 9$ were recombinantly expressed as human IgG1-isotype antibodies, and compared for their cross-reactivity profile, cross-neutralization capacity, mechanism of action, epitope characteristics and in vivo protective efficacy in subsequent experiments.

To determine the cross-reactivity of the $1.6 \mathrm{C} 7$ and $28 \mathrm{D} 9$ monoclonal antibodies we tested their binding to spike proteins of all five human-infecting betacoronaviruses and the HKU1related mouse hepatitis virus (MHV) by ELISA. The equimolar coating of the different antigens was verified by an antibody targeting the Strep-tag located at the C-termini of all antigens. 28D9-and to a lesser extent 1.6C7- displayed cross-reactivity and reacted with spike ectodomains of betacoronaviruses from different subgenera including MERS-CoV (subgenus Merbecovirus), SARS-CoV and SARS-CoV-2 (subgenus Sarbecovirus), and HCoV-OC43 and MHV (subgenus Embecovirus), whereas no reactivity was seen with the spike ectodomain $\left(\mathrm{S}_{\text {ecto }}\right)$ of $\mathrm{HCoV}$ HKU1 (subgenus Embecovirus) (Fig. 1a and Supplementary Fig. 2). Both mAbs bound equally well to MERS- $S_{\text {ecto }}$ and OC43$\mathrm{S}_{\text {ecto, }}$, shown by the ELISA-based half-maximal effective concentration $\left(\mathrm{EC}_{50}\right)$ values. Compared to $1.6 \mathrm{C} 7,28 \mathrm{D} 9$ was superior in its cross-reactivity profile, with stronger binding to SARS-S $S_{\text {ector }}$ SARS2-S ecto $_{\text {and }}$ MHV-S ecto $_{\text {(Fig. 1a). Both antibodies reacted to }}$ S2 ectodomains ( $S 2_{\text {ecto }}$ ) of MERS and MHV, indicating that their epitopes were located on the S2 fusion subunit of $\mathrm{CoV}$ spike proteins. 
a
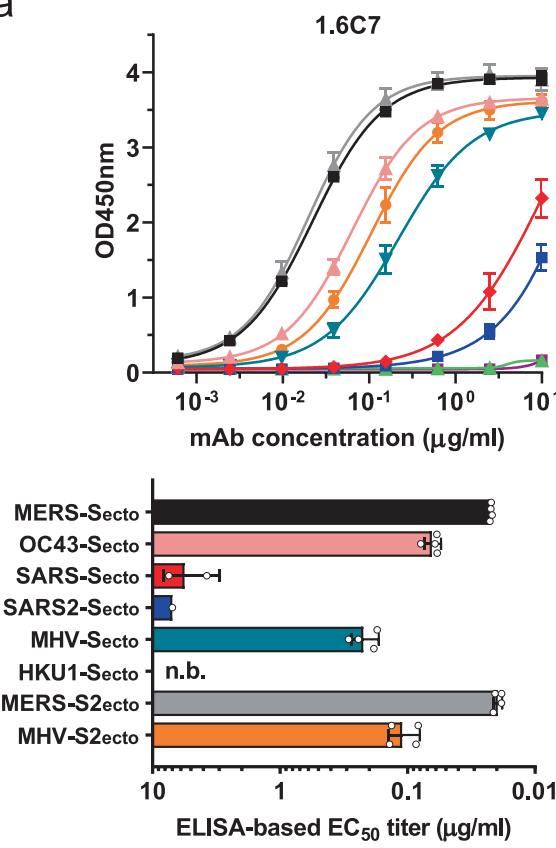

b

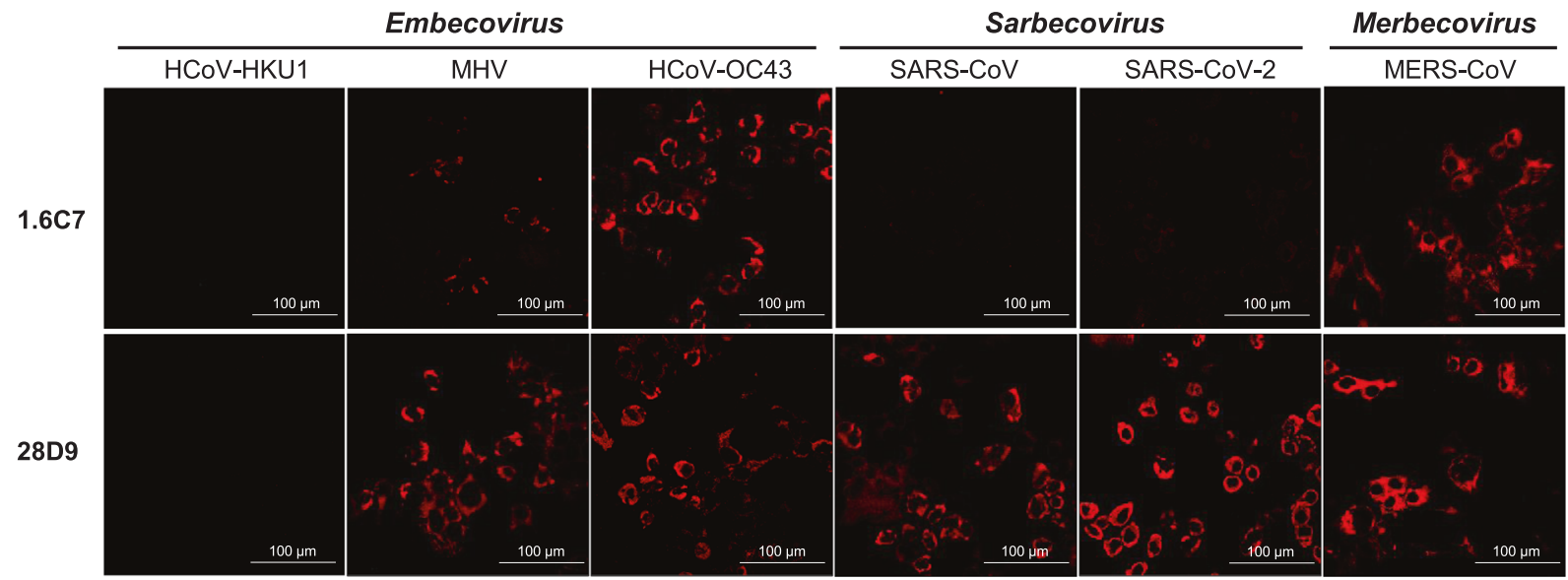

Fig. 1 Cross-reactivity of human monoclonal antibodies 28D9 and 1.6C7 to spike proteins of viruses in the Betacoronavirus genus. a ELISA binding curves (upper panels) and corresponding ELISA-based half-maximal effective concentrations ( EC $_{50}$ ) titers (lower panels) of mAbs $28 \mathrm{D} 9$ and $1.6 \mathrm{C} 7$ to Strep-tagged spike ectodomains $\left(\mathrm{S}_{\text {ecto }}\right.$ ) and S2 ectodomains $\left(\mathrm{S} 2_{\text {ecto }}\right.$ ) of betacoronaviruses from different subgenera including MERS-CoV (subgenus Merbecovirus), SARS-CoV and SARS-CoV-2 (subgenus Sarbecovirus), HCoV-OC43, HCoV-HKU1 and MHV (subgenus Embecovirus), coated at equimolar concentrations. Anti-strep mAb targeting the Strep-tagged antigens was used to corroborate equimolar plate coating. n.b., no binding. Graph bars represent the average \pm SD. Hollow circles represent individual data points for $n=2$ biological replicates and 2 technical replicates. Source data are provided as a Source Data file. b Binding of mAbs 1.6C7 and 28D9 to HEK-293T cells expressing GFP-tagged membrane-anchored full-length spike proteins of MERS-CoV, SARS-CoV, SARS-CoV-2, HCoV-OC43, HCoV-HKU1 and MHV detected by immunofluorescence assay. Cell nuclei in the overlay images were visualized by DAPI. The fluorescence images were recorded using a Leica Spell confocal microscope. Representative images from $n=2$ biological replicates. Scale bars are $100 \mu \mathrm{m}$.

Biolayer interferometry was used to characterize the binding kinetics and affinity of the cross-reactive antibodies to $\mathrm{CoV} \mathrm{S}$ ecto trimers immobilized on the surface of biosensors. The $1.6 \mathrm{C} 7 \mathrm{mAb}$ bound to the $\mathrm{S}_{\text {ecto }}$ of MERS-CoV, $\mathrm{HCoV}-\mathrm{OC} 43$ with equilibrium dissociation constants $\left(K_{D}\right)$ of 0.58 and $5.28 \mathrm{nM}$, respectively. Congruent with its ELISA-reactivity, $1.6 \mathrm{C} 7$ displayed low-affinity binding to the $\mathrm{S}$ ectodomains of SARS-CoV and SARS-CoV-2 with $K_{D}$ 's of 25.22 and $26.18 \mu \mathrm{M}$, respectively. $K_{D}$ 's of the 28D9 $\mathrm{mAb}$ with the MERS-, OC43-, SARS- and SARS2-S proteins were $0.72,7.45,3.17$ and $5.96 \mathrm{nM}$, respectively (Supplementary Fig. 3).

To assess whether the $1.6 \mathrm{C} 7$ and 28D9 antibodies could bind the full-length (i.c. membrane-anchored) version of spike proteins, we tested their reactivity to cells transiently expressing different (GFP-tagged) $\mathrm{CoV}$ spike proteins using immunofluoresence microscopy and flow cytometry. Binding of $1.6 \mathrm{C} 7$ was observed both in permeabilized and non-permeabilized cells expressing the spike proteins of $\mathrm{HCoV}-\mathrm{OC} 43$, MERS-CoV and MHV, whereas 28D9 additionally bound to cells expressing the spike proteins of SARS-CoV and SARS-CoV-2 (Fig. 1b, Supplementary Fig. 4 and Supplementary Table 1). No reactivity was seen for both antibodies to cells expressing the HCoV-HKU1 spike protein. These data demonstrate that both antibodies can react with full-length spike proteins, with specificities that are consistent with the CoV Secto ELISA reactivities. 
a
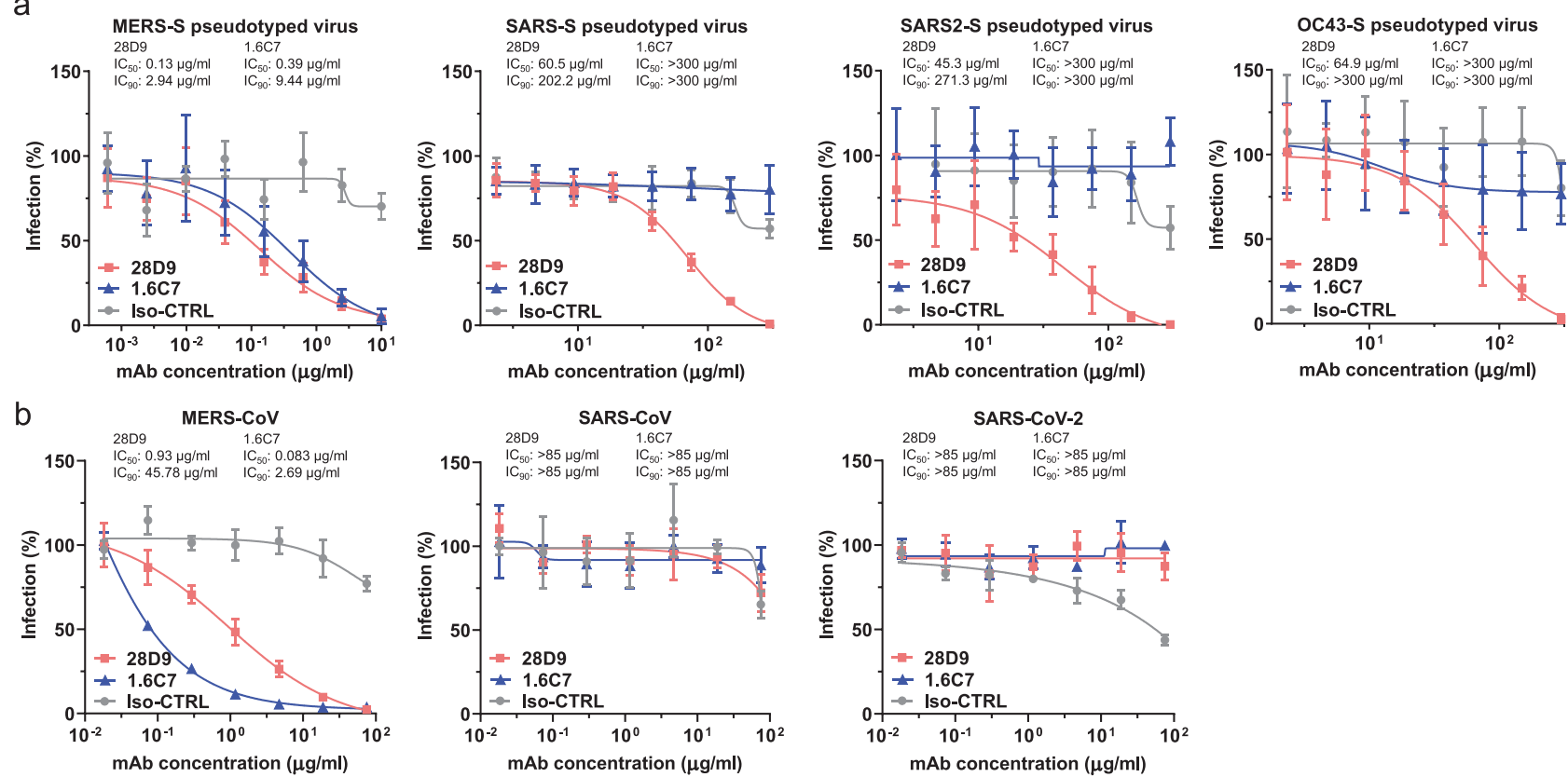

C

d
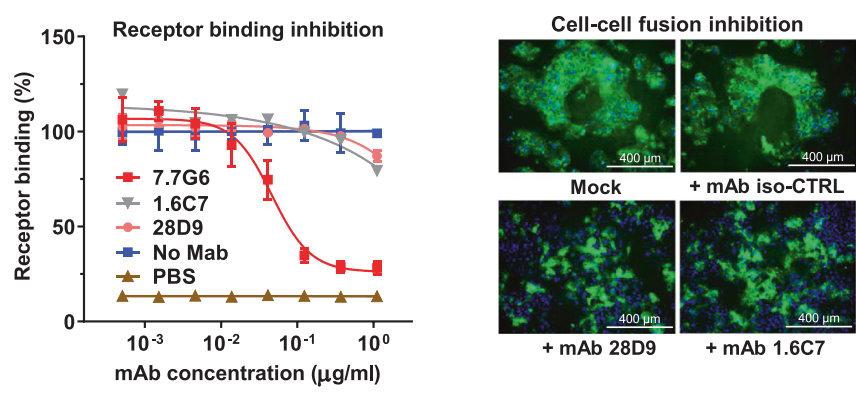

Fig. 2 Cross-neutralization capacity of mAbs 28D9 and 1.6C7 and mechanism of action. a Antibody-mediated neutralization of infection of luciferaseencoding VSV particles pseudotyped with spike proteins of MERS-CoV, SARS-CoV, SARS-CoV-2 and HCoV-OC43. Pseudotyped VSV particles preincubated with antibodies at indicated concentrations were used to infect VeroCCL81 cells (MERS-S pseudotyped VSV), VeroE6 cells (SARS-S and SARS2S pseudotyped VSV) or HRT-18 cells (OC43-S pseudotyped VSV) and luciferase activities in cell lysates were determined at $20 \mathrm{~h}$ post transduction to calculate infection (\%) relative to non-antibody-treated controls. The average \pm SD $(n \geq 6)$ from at least two independent experiments performed is shown. Iso-CTRL: an anti-Strep-tag human monoclonal antibody was used as an antibody isotype control. The $\mathrm{IC}_{50}$ and $\mathrm{IC}_{90}$ values were shown. Source data are provided as a Source Data file. b Antibody-mediated neutralization of MERS-CoV, SARS-CoV and SARS-CoV-2 infection. Neutralization of authentic viruses was performed using a plaque reduction neutralization test (PRNT) on VeroCCL81 cells (MERS-CoV) or VeroE6 (SARS-CoV and SARS-CoV-2) as described earlier ${ }^{74,75}$. The experiment was performed with triplicate samples, the average \pm SD is shown. The IC $C_{50}$ and IC $C_{90}$ values were shown. Source data are provided as a Source Data file. c ELISA-based receptor-binding inhibition assay. MERS-S ${ }_{\text {ecto }}$ pre-incubated with serially diluted mAbs was added to ELISA plates coated with soluble human DPP4. The binding of MERS-S ecto $_{\text {to }}$ DPP4 was detected using an HRP-conjugated antibody recognizing the Cterminal Strep-tag on MERS-S $S_{\text {ecto }}$. Data points represent the average $\pm S D$, for $n=3$ replicates from two independent experiments. Source data are provided as a Source Data file. d Cell-cell fusion inhibition assay. Huh-7 cells-transfected with plasmid expressing (GFP-tagged) MERS-CoV S were preincubated in the presence or absence of $1.6 \mathrm{C} 7$ and 28D9, or an irrelevant iso-type control antibody (Iso-CTRL), and then treated with trypsin to activate the membrane fusion function of the MERS-CoV S protein. The formation of MERS-S mediated syncytia was visualized by fluorescence microscopy. Merged images of MERS-S-GFP expressing cells (green) and DAPI-stained cell nuclei (blue) are shown. The experiment was performed twice, data from a representative experiment is shown. Source data are provided as a Source Data file.

Cross-neutralization capacity of mAbs 28D9 and 1.6C7 and mechanism of action. Next neutralizing activity by both antibodies against the targeted $\mathrm{HCoV}$ s was assessed. The $1.6 \mathrm{C} 7$ and 28D9 antibodies neutralized infection of MERS-S pseudotyped VSV ( $\mathrm{IC}_{50}$ values of 0.39 and $0.13 \mu \mathrm{g} / \mathrm{ml}$, respectively) as well as of authentic MERS-CoV ( $\mathrm{IC}_{50}: 0.083$ and $0.93 \mu \mathrm{g} / \mathrm{ml}$, respectively) (Fig. 2a, b). No neutralization was seen against authentic SARS$\mathrm{CoV}$ and SARS-CoV-2 by either of these antibodies, yet using the neutralization-sensitive VSV pseudoviral system ${ }^{34,44}$ low levels of cross-neutralizing activity by 28D9 were detected. 28D9 inhibited
OC43-S, SARS-S and SARS2-S pseudotyped VSV infection with $\mathrm{IC}_{50}$ values of $64.9,60.5$ and $45.3 \mu \mathrm{g} / \mathrm{ml}$, respectively (Fig. 2a). To understand the mechanism of virus neutralization, we assessed antibody interference with spike-mediated receptor-binding and membrane fusion activity. In line with our earlier observations for $1.6 C 7^{43}$, we found that 28D9 inhibits MERS-S driven cell-cell

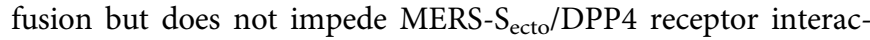
tion (Fig. 2c, d), suggesting that both S2-targeting antibodies prevent the membrane fusion function of the spike S2 subunit that is required for infection. 
a

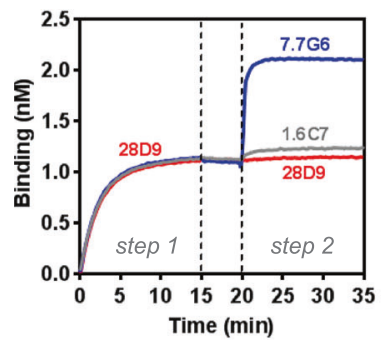

b

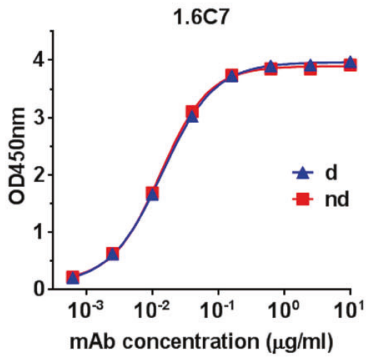

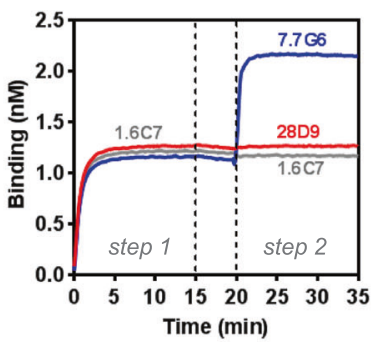

28D9

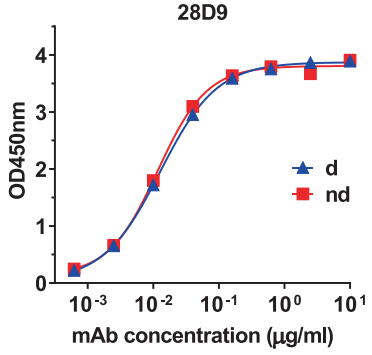

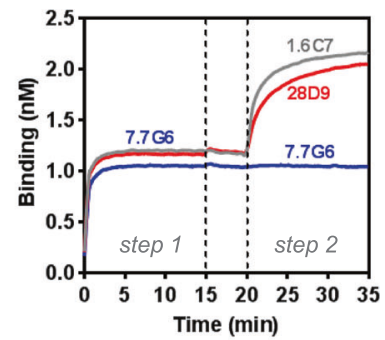
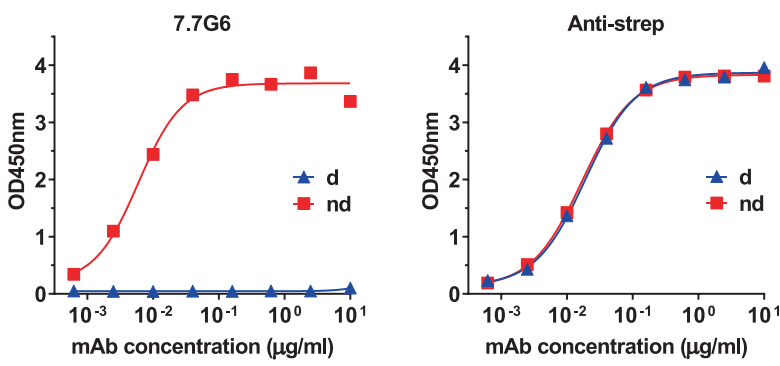

C

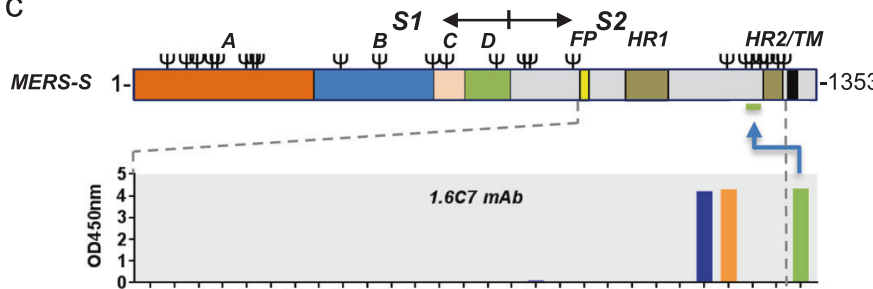

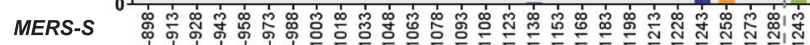

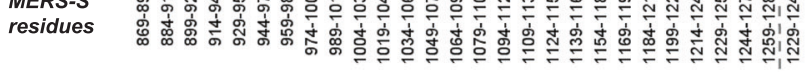

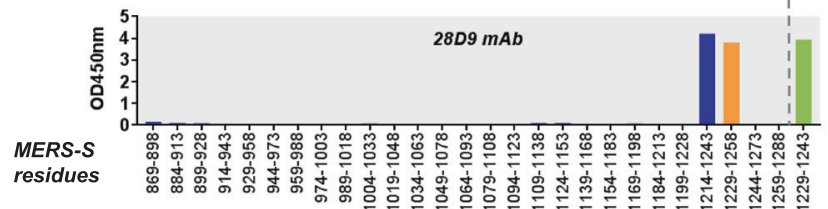

d
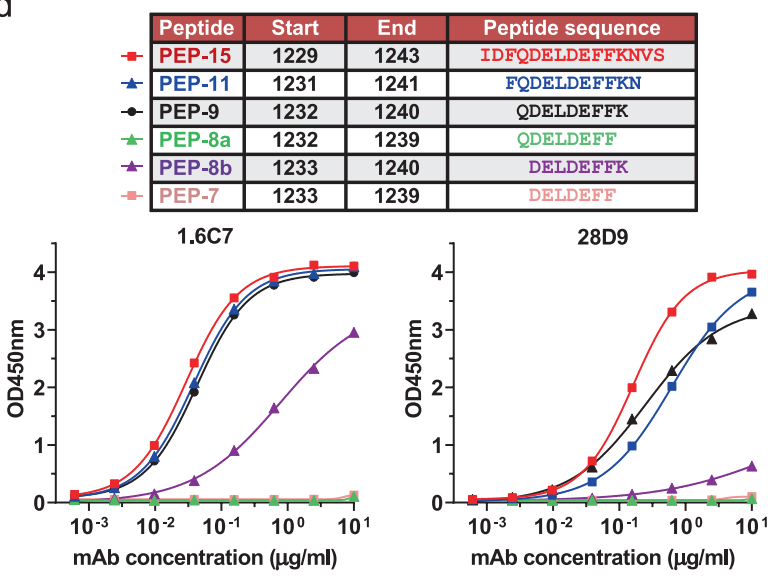

Fig. 3 mAbs 1.6C7 and 28D9 target a linear epitope located in the stem region of S2 fusion subunit. a Antibody binding competition analysed by biolayer

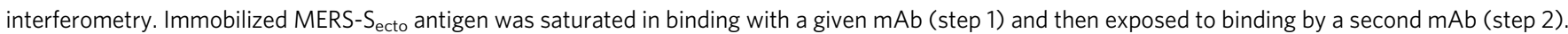
Additional binding of the second antibody indicates the presence of an unoccupied epitope, whereas lack of binding indicates epitope blocking by mAb1. As a control, the first $\mathrm{mAb}$ was also included in the second step to check for self-competition. The competitive binding was tested for the S2-targeting 1.6C7 and 28D9 antibodies and a MERS-S1 antibody control (7.7G6) ${ }^{43}$. Source data are provided as a Source Data file. b $1.6 \mathrm{C} 7$ and $28 \mathrm{D} 9$ recognize a linear epitope. ELISA binding curves of 1.6C7 and 28D9 to untreated MERS-S ${ }_{\text {ecto }}$ (non-denatured: 'nd') versus MERS-S ecto $_{\text {that }}$ was heat-denatured in the presence of SDS and DTT (denatured: ' $d$ '). Two antibodies targeting the MERS-S1 domain (7.7G6) and the 8-residue long linear Strep-tag epitope (antistrep) were used as controls. Source data are provided as a Source Data file. c 1.6C7/28D9 epitope maps to a 15-aa long stem region upstream of HR2 in MERS-S. ELISA-reactivity of 1.6C7 and 28D9 to a peptide library of 30-amino acid long peptides (with 15-a.a. overlap) covering the conserved C-terminal part of the MERS- $S_{\text {ecto }}$ (residues 869-1,288). Both antibodies reacted with two peptides (blue and orange bars), and with a peptide corresponding to their 15-a.a. long overlap (green bar; MERS-S residues 1,229-1,243). The position of the epitope containing region is indicated in the MERS-S protein schematic with the spike subunits (S1 and S2), S1 domains (A through D), fusion peptide (FP), heptad repeat 1 (HR1), heptad repeat 2 (HR2) and transmembrane domain (TM) annotated. Source data are provided as a Source Data file. $\mathbf{d}$ The 1.6C7/28D9 epitope maps to a 8-aa long peptide 'DELDEFFK' detected by ELISA. ELISA binding curves of $1.6 \mathrm{C} 7$ and $28 \mathrm{D} 9$ to $\mathrm{N}$ - and C-terminally truncated versions of the 15-mer peptide fragment of MERS-S. Data points represent the average from $n=2$ technical replicates. Source data are provided as a Source Data file.

mAbs 1.6C7 and 28D9 target a linear epitope in the stem region of the $\mathbf{S} 2$ fusion subunit. Both antibodies were tested for competitive binding to MERS- $S_{\text {ecto }}$ using biolayer interferometry. MERS-S $S_{\text {ecto }}$ binding by $1.6 \mathrm{C} 7$ antibody completely prevented binding of 28D9, and vice versa, suggesting that the antibodies target overlapping epitopes (Fig. 3a). To assess the epitope conformational specificity, we compared the ELISA reactivity of $1.6 \mathrm{C} 7$ and 28D9 to untreated MERS-CoV $\mathrm{S}_{\text {ecto }}$ antigen versus antigen that was heat-denatured in the presence of SDS and DTT. Both antibodies reacted equally well with non-denatured and denatured proteins, unlike a MERS-S1 targeting antibody $7.7 \mathrm{G} 6^{43}$ that only reacted with non-denatured antigen. These data indicate that 1.6C7 and 28D9 likely bind a linear, contiguous sequence of amino acids in MERS-S (Fig. 3b).

As both antibodies may bind a linear epitope, we aimed to map their epitope location by ELISA using an array of overlapping synthetic peptides (30-mer peptides with an overlap of 15 residues) covering the conserved C-terminal part of the MERS-

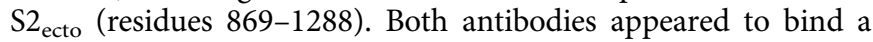
15-residue-long region (MERS-S residues 1229-1243) positioned just upstream of the heptad repeat 2 (HR2) region in the MERSS2 fusion subunit (Fig. 3c). Analysis of 1.6C7/28D9 antibody binding to $\mathrm{N}$ - and C-terminally truncated versions of the 15 -mer peptide fragment designated the $\mathrm{D}^{1233}$ ELDEFFK $^{1240}$ MERS-S 
a

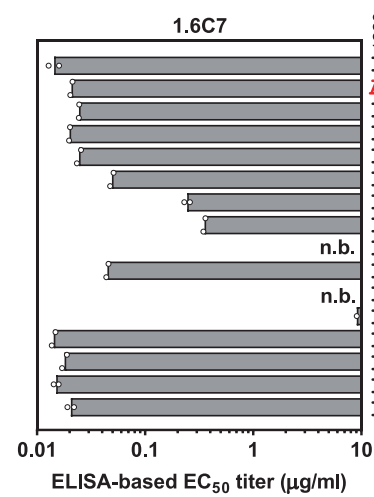

C

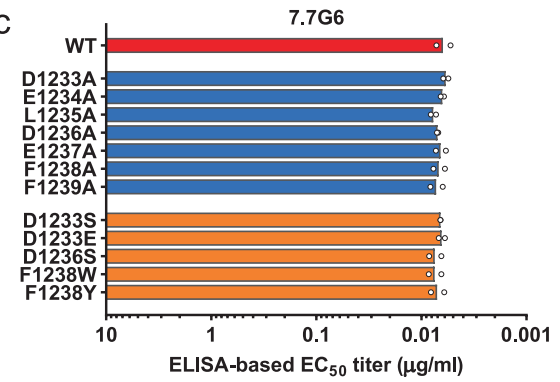

d

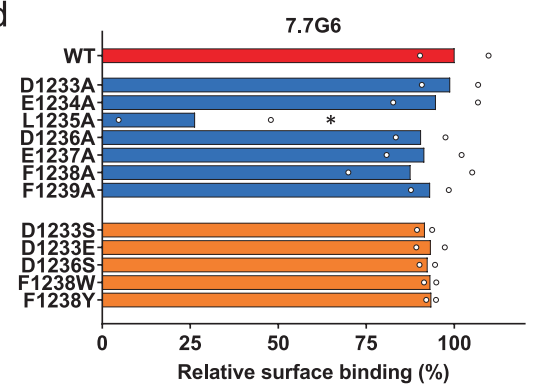

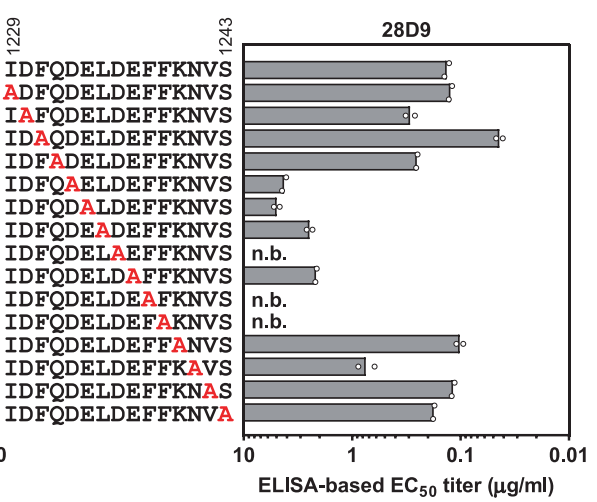
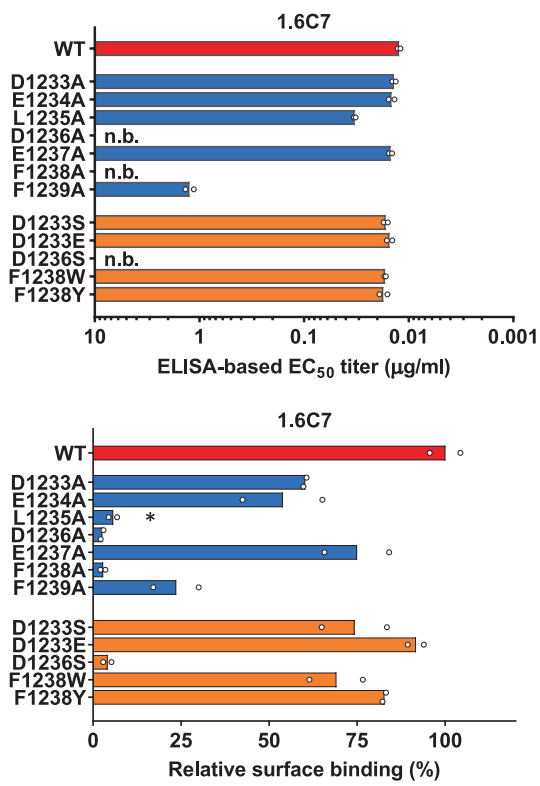

b

\begin{tabular}{|c|c|c|c|c|}
\hline MERS & & $\Xi$ & 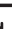 & $\mathrm{K}$ \\
\hline OC43 & $E$ & . & & \\
\hline SARS & $\mathrm{E}$ & • & & \\
\hline SARS2 & $E$ & . & & \\
\hline MHV & $E$ & . & & \\
\hline HKU1 & $\mathrm{S}$ & & & \\
\hline
\end{tabular}
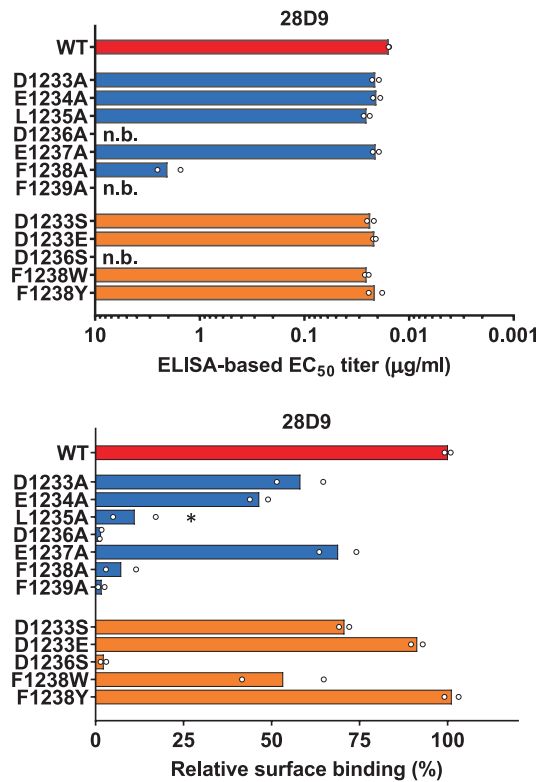

Fig. 4 Fine mapping the 1.6C7 and 28D9 antibody binding sites on the spike protein by mutagenesis. a ELISA-based epitope alanine mutagenesis on the 15 -mer spike peptide fragment comprising the linear $1.6 \mathrm{C} 7$ and 28D9 epitope, shown by half-maximum effective concentration (EC 50 ) titers $(\mu \mathrm{g} / \mathrm{ml})$. The average from two independent experiments performed is shown. Source data are provided as a Source Data file. b Sequence alignment of 1.6C7/28D9 epitope region of MERS-CoV, HCoV-OC43, SARS-CoV, SARS-CoV-2, MHV and HCoV-HKU1. c Spike protein ectodomain single site mutagenesis to delineate $1.6 \mathrm{C} 7$ and 28D9 antibody binding sites. ELISA-based $\mathrm{EC}_{50}$ titers $(\mu \mathrm{g} / \mathrm{ml})$ of 1.6C7/28D9 binding to MERS-S $\mathrm{S}_{\text {ecto }}$ mutants containing single amino acid substitutions in the core epitope region are indicated on the left. Anti-MERS-S1 control antibody 7.7G6 was used to control the expression level of all mutants. n.b., no binding. The average from $n=2$ biological replicates is shown. Source data are provided as a Source Data file. d Binding of 28D9 and $1.6 \mathrm{C} 7$ antibodies to cell surface expressing (GFP-tagged) MERS-S mutants containing single amino acid substitutions in the core epitope region detected by flow cytometry. Antibody binding was detected using AlexaFluor 594 conjugated secondary antibody. The relative surface binding was determined by calculating the percentage of GFP+/Alexa Fluor $594^{+}$cells over GFP+ cells. Anti-MERS S1 antibody $7.7 \mathrm{G} 6$ was used to control the cell surface expression levels of all single-site mutants. The asterisk indicates reduced cell surface expression of the L1235A mutant. n.b., no binding. The average from $n=2$ biological replicates is shown. Source data are provided as a Source Data file.

protein segment as the minimal epitope region still affording some binding by both antibodies (Fig. 3d).

Fine mapping the mAbs binding sites on the spike protein by mutagenesis. To map residues critical for binding by the $1.6 \mathrm{C} 7 /$ 28D9 antibodies, we performed ELISA-based epitope alanine scanning mutagenesis on the 15-mer spike peptide fragment comprising the linear epitope. The alanine scanning analysis of the 15 -mer peptide fragment defined three residues $\left(\mathrm{D}^{1236}, \mathrm{~F}^{1238}\right.$ and $\mathrm{F}^{1239}$ ) as critical to binding by both antibodies (Fig. $4 \mathrm{a}$ and Supplementary Fig. 5), with another four residues $\left(D^{1233}, E^{1234}\right.$, $\mathrm{L}^{1235}$ and $\mathrm{E}^{1237}$ ) that contribute to binding by 28D9 and-to a lesser extent-by 1.6C7. To corroborate the epitope alanine scanning data, we assessed ELISA reactivity of the 1.6C7/28D9 antibodies to single-site MERS- $S_{\text {ecto }}$ mutants containing alanine substitutions in the core epitope region. Consistent to the peptide alanine scanning, alanine substitution of the three residues $\mathrm{D}^{1236}$, $\mathrm{F}^{1238}$ and $\mathrm{F}^{1239}$ abrogated MERS-S $\mathrm{S}_{\text {ecto }}$ binding by both antibodies, but not that of the anti-MERS-S1 control antibody 7.7G6 (Fig. 4c).

To assess the individual contribution to antibody binding of identified residues in the context of the membrane-anchored fulllength spike protein, a flow cytometry-based assay was performed measuring antibody binding to cell-surface expressed MERS-S mutants (Fig. 4d). The anti-MERS-S1 7.7G6 antibody was used to control cell surface expression levels of the single-site mutants. With the exception of the $\mathrm{L}^{1235} \mathrm{~A}$ mutant, all mutant spike proteins displayed surface expression levels similar to wildtype. Alanine substitutions of residues in the MERS-S core epitope region $\left(D^{1233}\right.$ ELDEFFK $\left.^{1240}\right)$ reduced binding by both antibodies to a varying extent, although analysis of binding data for the 
$\mathrm{L}^{1235} \mathrm{~A}$ mutant was compromised by reduced cell surface expression level. Consistent with the ELISA-based analysis (Fig. $4 \mathrm{a}, \mathrm{c}$ ), mutation of $\mathrm{D}^{1236}, \mathrm{~F}^{1238}$ and $\mathrm{F}^{1239}$ most strongly affected binding by both antibodies. Both antibodies showed similar binding patterns in all three antigen-binding assays (Fig. 4a, c, d and Supplementary Fig. 5), yet subtle differences in the reactivities were observed that may rationalize differences between these two antibodies and their cross-spike binding properties.

The identified residues in the spike epitope region $\left(\mathrm{D}^{1233} \mathrm{ELDEFFK}^{1240}\right.$ in MERS-S) contributing to $1.6 \mathrm{C} 7 / 28 \mathrm{D} 9$ binding are not fully conserved in the targeted betacoronavirus spike proteins, with variations found in three residues $\left(\mathrm{D}^{1233}\right.$, $\mathrm{D}^{1236}$ and $\mathrm{F}^{1238}$ in MERS-S) that are key to binding by both cross-reactive antibodies (Fig. $4 \mathrm{~b}$ ). To assess the contribution of these amino acid variations to antibody reactivity, single-site substitutions were made in MERS-S. Soluble and full-length $\mathrm{D}^{1233} \mathrm{~S}, \mathrm{D}^{1233} \mathrm{E}, \mathrm{D}^{1236} \mathrm{~S}, \mathrm{~F}^{1238} \mathrm{~W}$ and $\mathrm{F}^{1238} \mathrm{Y}$ MERS-S mutants were tested for antibody binding by respectively ELISA (Fig. 4c) and flow cytometry (Fig. 4d), as described above. Substitution of $\mathrm{D}^{1233}$ in MERS-S to serine (S, found in HKU1-S) or glutamic acid (E, found in SARS-, SARS2-, OC43- and MHV-S) still allowed efficient binding by both antibodies. In addition, but contrary to the MERS-S F ${ }^{1238} \mathrm{~A}$ mutant, the MERS-S mutants in which $\mathrm{F}^{1238}$ was replaced by a tryptophan (W, found in HKU1-, OC43- and MHV-S) or tyrosine ( $\mathrm{Y}$, found in SARS- and SARS2-S) maintained antibody binding reactivity. However, the reciprocal substitution in SARS-S ( $\mathrm{Y}^{1137} \mathrm{~F}$ in SARS-S numbering) resulted in a strong increase in binding by $1.6 \mathrm{C} 7$ to SARS-S. In contrast, this substitution did not alter ELISA-binding activity by 28D9 (Supplementary Fig. 6). These data indicate that a certain degree of sequence variability is allowed in the epitope region without compromising antibody binding, with slight differences between 28D9 and 1.6C7. Particularly for 28D9 that shows the broadest reactivity, different aromatic residues (Phe, Trp and Tyr) seem to be permitted at MERS-S residue position 1238. Conversely, the $\mathrm{D}^{1236} \mathrm{~S}$ substitution in MERS-S (found in HKU1-S) fully abrogated binding by both antibodies. The reciprocal substitution in HKU1-S ( $\left({ }^{1239} \mathrm{D}\right.$ in HKU1-S numbering) was sufficient to rescue binding by 28D9, and to a lesser extend by $1.6 \mathrm{C} 7$ (Supplementary Fig. 7). These data rationalize the observed binding activities of both antibodies to spike proteins of MERS$\mathrm{CoV}$, SARS-CoV, SARS-CoV-2, HCoV-OC43 and MHV, as well as the inability of both antibodies to bind the HCoV-HKU1 spike protein. Evidently, epitope mapping of the two antibodies raised in two different animal immunization experiments revealed that they target the same epitope. We identified a third cross-reactive monoclonal antibody from an independent mouse immunization experiment (DNA + protein immunization), that targets the same site on betacoronavirus spike proteins (mAb 18H2, Supplementary Fig. 8), indicating that this epitope is immunogenic and efficient in the induction of cross-reactive antibodies.

Sequence analysis of the variable regions of the 1.6C7, 28D9 and $18 \mathrm{H} 2$ antibodies identified that their heavy and light chains were derived from the same IGHV6-1 and IGKV4-1 germline precursor, respectively (Supplementary Fig. 9). As these antibodies were obtained from three different immunization campaigns and thus not clonally related, this suggests epitope-dependent preferential germline usage and VH/VL pairing and germlineencoded affinity for cognate antigen as reported previously for other epitopes and viruses ${ }^{45-50}$. The antibody heavy and light chains sequences deviate in the CDR3 region $(\mathrm{V} / \mathrm{D} / \mathrm{J} ; \mathrm{V} / \mathrm{J})$ and were somatically mutated. Seven somatic hypermutations resulting in amino acid changes were found in the $\mathrm{VH}$ region for 28D9, and six for 1.6C7. Seven somatic hypermutations were found in the VL region for 28D9, and two for 1.6C7.
Glycans on antigens are frequently found to be a determinant for antibody binding. We found that antigen deglycosylation resulted in lower binding of 28D9 to OC43-S $\mathrm{S}_{\text {ecto }}, \mathrm{MHV}-\mathrm{S}_{\text {ecto }}$ and SARS- $S_{\text {ecto }}$ using western blotting (Supplementary Fig. 10a), in contrast to $1.6 \mathrm{C} 7$. We tested the involvement of an $\mathrm{N}$-linked glycan located one residue downstream of the core epitope region $\left(\mathrm{D}^{1233} \mathrm{ELDEFFK}^{1240}\right)$ in MERS-S. This N-glycosylation sequon $(\mathrm{NxS} / \mathrm{T})$ is conserved among betacoronavirus spike protein orthologous (Fig. 5a). Deletion of the $\mathrm{N}^{1241}$ glycosylation site in MERS- $S_{\text {ecto }}$ did not impair binding by both antibodies in ELISA (Supplementary Fig. 10b). In contrast, deletion of the orthologous glycosylation site in OC43-S $\mathrm{S}_{\text {ecto }}\left(\mathrm{N}^{1243}\right.$ in OC43-S numbering) fully abrogated ELISA-reactivity of 28D9, whereas that of 1.6C7 was virtually unchanged. These data point towards the involvement of an N-linked glycan in antigen binding by 28D9, however a loss of epitope conformation integrity inflicted by the glycan elimination cannot be excluded as an alternative explanation.

1.6C7 and 28D9 target the stem helix of the coronavirus spike protein. Although the 28D9/1.6C7 epitope region was present in five betacoronavirus spike protein ectodomains of which cryo-EM prefusion structures were elucidated, the C-terminally located epitope region was not or only poorly resolved in the cryo-EM maps, indicative for conformational flexibility of the epitope region $22,32,51$. However, the epitope was recently revealed in trimeric pre- and postfusion SARS-CoV-2 spike structures that were reconstructed by cryo-EM using purified full-length spike protein $^{31}$. In the prefusion SARS-CoV-2 spike, the epitope region $\left(\mathrm{E}^{1150} \mathrm{ELDKYFK}^{1157}\right.$ in SARS2-S) is part of a 20-residue-long ahelix at the membrane-proximal base of the molecule (Fig. 5b). The corresponding region in $\mathrm{HCoV}-\mathrm{NL63}$ spike protein was designated as 'stem' helix ${ }^{51}$. The stem helices of three $\mathrm{S}$ protomers align along the three-fold symmetry axis and form a helical bundle accessible for antibody binding, and which displays a relatively high level of conserved surface-exposed residues across betacoronavirus spike proteins (Supplementary Fig. 11). Remarkably, both antibodies can also bind the postfusion spike structure as demonstrated by their ability to bind the MHV S2 2 ecto (Fig. 1a) that was earlier solved in its postfusion structure ${ }^{52}$. In the $\mathrm{S} 2$ postfusion structure, the $\mathrm{N}$ - and $\mathrm{C}$-terminal residues of the stem helix are uncoiled whereas the middle part $\left(\mathrm{F}^{1148} \mathrm{KEELD}^{1153}\right.$ in SARS2-S) is still folded as a short, surface-exposed helix, which docks perpendicular to the central coiled coil ${ }^{31,52}$

Antibodies towards the stem helix epitope are elicited during natural infection. We next analysed whether antibodies towards the stem helix epitope or other linear epitopes in the spike protein are elicited during natural MERS-CoV infection in humans and dromedary camels. Spike protein peptide microarray analysis with five human and four dromedary camel MERS-CoV-positive sera on 905 overlapping peptides covering the entire spike protein ectodomain (MERS-S residues 1-1296) identified sixteen linear core epitopes (Fig. 6 and Supplementary Fig. 12). Five of them were concentrated in the \pm 80 -residue long region upstream of the spike transmembrane domain comprising the HR2 region, which undergoes extensive structural rearrangements during fusion. One of these five peptides ( $\mathrm{D}^{1230}$ FQDELDEFFKNVS ${ }^{1243}$ ) is overlapping the epitope core region DELDEFFK of the $1.6 \mathrm{C} 7 /$ 28D9 antibodies (Fig. 6c) and is-compared to other identified linear peptide epitopes-recognized most frequently by MERSpositive sera from humans (3/5) and dromedary camels $(2 / 4)$ (Fig. 6c and Supplementary Fig. 12). These data indicate that antibodies towards this epitope are efficiently elicited not only in spike-immunized mice but also during natural infection of humans and dromedary camels. 
a
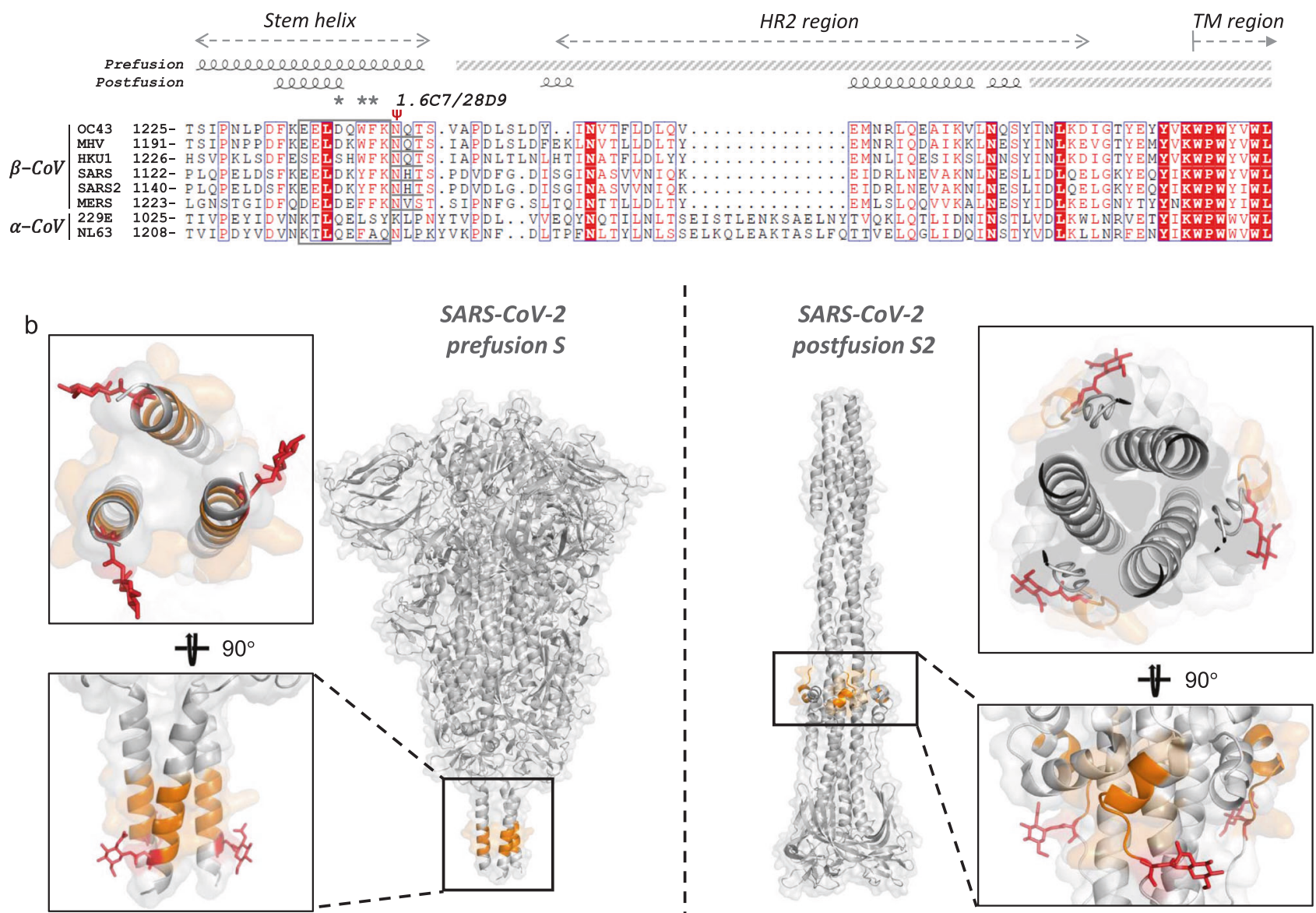

Fig. 5 1.6C7 and 28D9 bind the membrane-proximal stem helix of the coronavirus spike protein. a Sequence alignment of spike protein region of alphaand betacoronaviruses encompassing the 1.6C7/28D9 epitope region using EMBL-EBI Clustal Omega programme (https://www.ebi.ac.uk/Tools/msa/ clustalo/). The 28D9 and 1.6C7 core epitope region is outlined by a rectangle box and residues critical for antibody binding are annotated by asterisks. A conserved glycosylation sequon ( NxS/T) found in betacoronavirus spike proteins-one amino acid downstream of the core epitope-is underlined and annotated $(\psi)$. The stem helix, heptad repeat region 2 (HR2) and the start of the transmembrane domain (TM) are indicated. Secondary structural elements of the SARS-CoV-2 prefusion spike (PDB: 6XR8) and postfusion S2 (PDB: 6XRA) structures are visualized using ESPript 3.0 (http://espript.ibcp. $\mathrm{fr} /$ ESPript/ESPript/). b Structures of the SARS-CoV-2 spike (PBD: 6XR8) and S2 (PDB: 6XRA) in pre- and postfusion conformation, respectively. Structures are indicated as a grey cartoon with transparent surface presentation, and the segment corresponding to the stem helix epitope coloured in orange. Insets: zoom-in sections of the epitope region in both structures in two different orientations with the conserved $\mathrm{N}$-glycan highlighted in red.

Antibody-mediated protection of mice challenged with MERSCoV or SARS-CoV. To assess the in vivo protection capacity of antibodies targeting the stem-helix epitope, we tested the prophylactic and therapeutic activity of the $1.6 \mathrm{C} 7 \mathrm{mAb}$ against lethal MERS-CoV challenge using the K18-hDPP4 transgenic mouse model expressing human DPP4 ${ }^{53}$. 20-30-week-old mice were injected with $50 \mu \mathrm{g}$ of antibody (equivalent to $1.8 \mathrm{mg} \mathrm{mAb}$ per $\mathrm{kg}$ body weight) by intraperitoneal injection $24 \mathrm{~h}$ before (pre-) or $24 \mathrm{~h}$ after infection (post-) with a lethal dose of MERS-CoV. A highly potent MERS-CoV neutralizing antibody 7.7G6, and an irrelevant IgG1 isotype control antibody ${ }^{43}$ were taken along as controls (Fig. 7a). The percentage of survival and weight change was monitored daily for 10 days. In isotype control pre- and posttreated mice, MERS-CoV causes lethal disease with $100 \%$ lethality between 8 and 10 days and showed significant weight loss. In contrast, both pre- and post-treatment of $1.6 \mathrm{C} 7$ protected mice from death, and that of $7.7 \mathrm{G} 6$ protect $80-100 \%$ of mice from death. Relative to isotype control-treated mice, mice treated with either 7.7G6 or 1.6C7 showed reduced weight loss (Fig. 7a).

To investigate the reduction in pathology and viral loads, lungs of mice were harvested on day 3 (three animals) and 8-10 (five animals) post infection. In isotype control-treated mice, no obvious difference of viral RNA was observed compared to nonantibody treated (Mock-treated) mice. However, mice treated with 1.6C7 before or after virus challenge, demonstrated 1-2 log reduction in viral RNA titers on day 3 post exposure, whereas a 2-3 log reduction was seen at 8-10 dpi (Supplementary Fig. 13a). A similar reduction was seen for the isolation of infectious virus at day 3, whereas at day 8-10 post exposure no infectious virus could be isolated from the lungs of the $1.6 \mathrm{C} 7$ pre- and postexposure treated mice (Supplementary Fig. 13a). Mice treated with 1.6C7 before and after infection showed greatly reduced lung pathology and inflammation following MERS-CoV infection at day 3 and 8-10, consistent with viral RNA levels and virus titers determined for these mice (Supplementary Fig. 13b).

We next tested the in vivo protection activity of the 28D9 mAb against MERS-CoV and SARS-CoV challenge. Mice $(n=5)$ were injected with two doses (50 or $200 \mu \mathrm{g}$ ) of 28D9 or an isotype control antibody by intraperitoneal injection $24 \mathrm{~h}$ before intranasal infection with a lethal dose of MERS-CoV or of SARS-CoV (Fig. 7b, c), respectively. The percentage of survival and weight change of mice was monitored daily for 13 days. Whereas no protection was seen against SARS-CoV challenge, four out of five animals infused with $200 \mu \mathrm{g} 28 \mathrm{D} 9$ antibody 
a

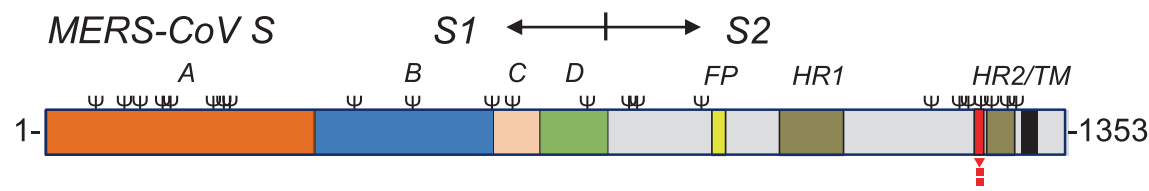

b

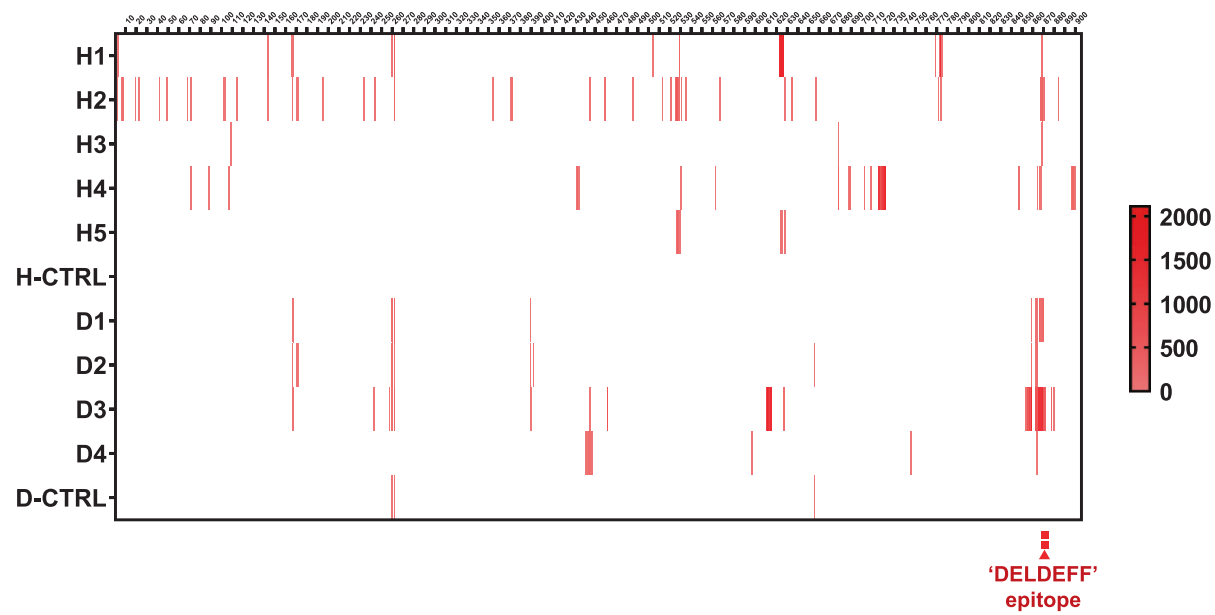

C

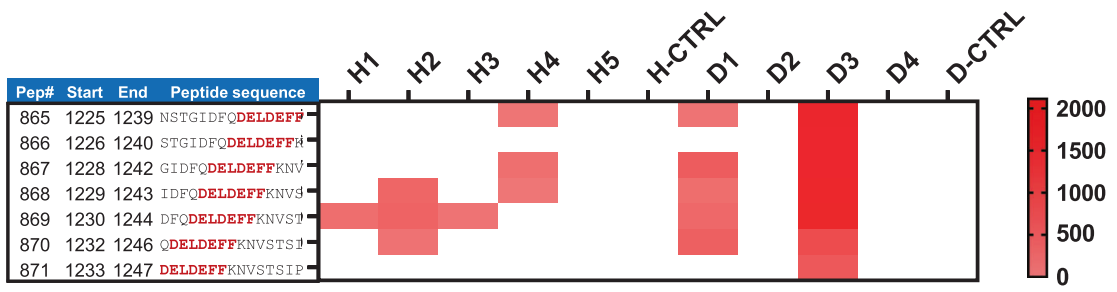

Fig. 6 Antibodies towards the stem helix epitope are elicited during natural infection. a Schematic representation of the MERS-CoV spike protein. The spike subunits (S1 and S2), S1 domains (A through D), fusion peptide (FP), heptad repeat 1 (HR1), heptad repeat 2 (HR2) and transmembrane domain (TM) are annotated. b Spike protein peptide microarray analysis using MERS-positive human and dromedary camel sera. 905 overlapping peptides covering the entire MERS-CoV S ectodomain (residues 1-1296) were synthesized with an offset of one or two residues. The binding of five convalescent MERS-positive human $(\mathrm{H} 1-\mathrm{H} 5)$ and four dromedary camel (D1-D4) sera to the peptide library, as well as a MERS-negative serum from human (H-CTRL) or camel (DCTRL) was assessed in a PEPSCAN-based ELISA (Lelystad, The Netherlands). A cumulative heatmap of signal intensities for individual peptides are shown. Signal intensities increase from light reddish to red, whereas white corresponds to background signal. Source data are provided as a Source Data file. c Reactivity of the human and dromedary sera to peptides covering the 1.6C7/28D9 epitope region (epitope core sequence highlighted in red). Source data are provided as a Source Data file.

survived MERS-CoV infection and did not display significant weight loss.

\section{Discussion}

We report two human monoclonal antibodies that are able to bind the spike proteins of betacoronaviruses from distinct subgenera. Both antibodies were found to target a conserved and immunogenic epitope in the membrane-proximal stem region on the S2 fusion subunit, that is also recognized by the immune system during natural infection. Antibodies targeting this antigenic site prevented mortality and disease in mice upon MERS$\mathrm{CoV}$ infection, demonstrating the relevance of the epitope for providing protection.

The cross-binding activity of in particular 28D9 towards five betacoronaviruses (MHV, HCoV-OC43, MERS-CoV, SARS-CoV and SARS-CoV-2) was remarkable since the targeted spike protein sequences are highly divergent and share only about $15 \%$ overall protein sequence identity with $26 \%$ identity across the more conserved S2 fusion subunit. Three residues in the stem helix epitope region appeared to be critical for antibody binding. Two of those appeared fully conserved in the targeted betacoronavirus spike sequences ( $\mathrm{D}^{1236}$ and $\mathrm{F}^{1239}$ in MERS-S), whereas only aromatic amino acids residues were found at the third position (F1238 in MERS-S), indicative of functional conservation. The location of the epitope was corroborated by the gain-of-function binding for the HCoV-HKU1 spike protein upon serine substitution at MERS-S equivalent position $\mathrm{D}^{1236}$. The small number of (functionally) conserved key residues targeted by both antibodies and the involvement of a conserved Nlinked glycan may rationalize their binding to these highly divergent antigens $s^{54,55}$. While our extensive biochemical and mutagenesis studies give insight into the epitope position and epitope residues critical for antibody binding, structural analysis of antibody-spike complex is needed to provide a detailed molecular understanding for broad-spectrum activity.

We showed that the stem-helix targeting antibodies effectively blocked MERS-CoV infection in cultured cells and provided prophylactic and therapeutic protection to mice against a lethal dose MERS-CoV challenge. This epitope was hitherto not described as a target of neutralizing antibodies, although monoclonal antibodies with neutralizing activity were found to target the sequences flanking the stem-helix epitope, including the downstream HR2 region ${ }^{56-58}$. Notably, no amino acid 
a

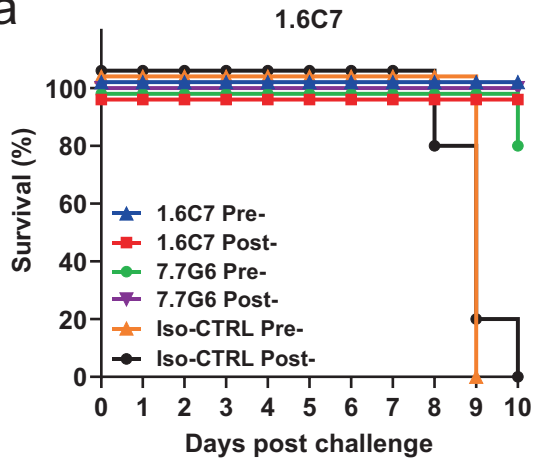

b

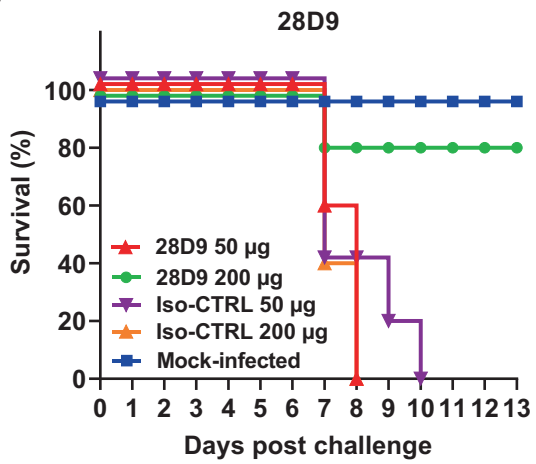

C

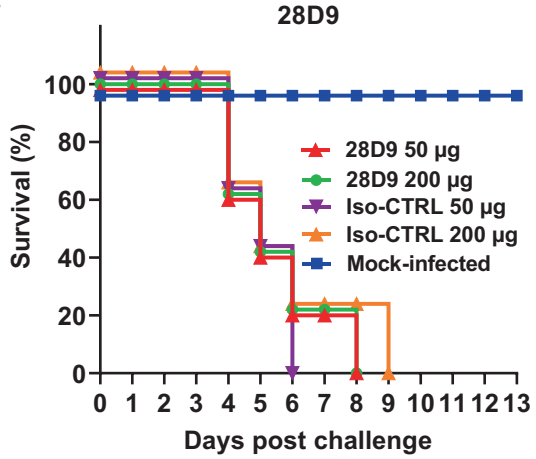

$1.6 \mathrm{C} 7$

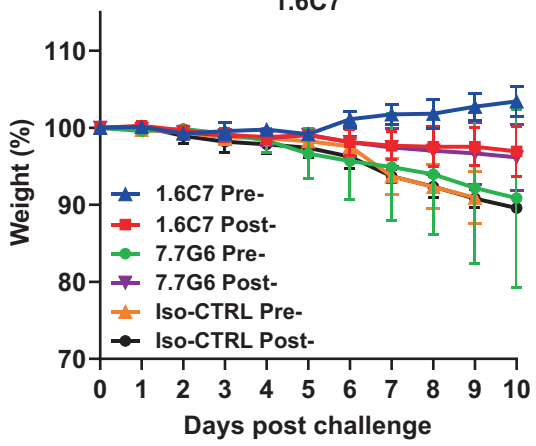

28D9

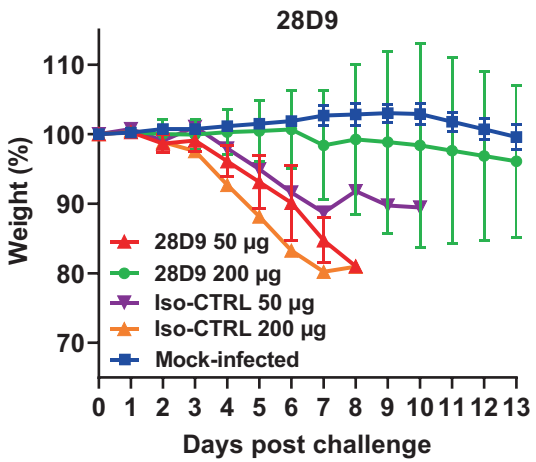

28D9

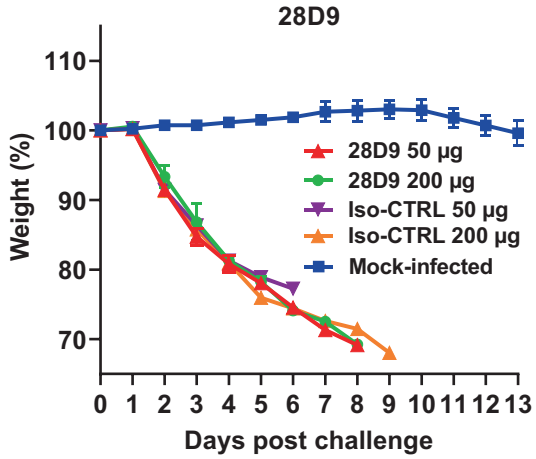

Fig. 7 Antibody-mediated protection of mice against lethal MERS-CoV/SARS-CoV challenge. a The in vivo prophylactic and therapeutic activity of the $1.6 \mathrm{C} 7 \mathrm{mAb}$ against lethal dose MERS-CoV challenge was tested in the K18 transgenic mouse model expressing human DPP4 ${ }^{53}$. A potent neutralizing MERS-S1 antibody (7.7G6) or an irrelevant lgG1 control antibody was taken along. Eight 20-30-week-old mice were injected with $50 \mu g$ of antibody (equivalent to $1.8 \mathrm{mg} \mathrm{mAb} / \mathrm{kg}$ body weight) by intraperitoneal injection $24 \mathrm{~h}$ before (pre-) or $24 \mathrm{~h}$ after (post-) intranasal infection with a lethal dose of MERS-CoV. Survival rates (left) and weight loss (right, expressed as a percentage of the initial weight) were monitored daily until 10 days post-inoculation. Data points represent mean body weight relative to the initial weight $\pm S D, n=8$ mice. Source data are provided as a Source Data file. $\mathbf{b}$ Prophylactic efficacy of 28D9 against MERS-CoV infection. Five 20-week-old K18 mice were mock-infected or injected with 50 or $200 \mu \mathrm{g}$ of 28D9 (equivalent to 1.8/ $7.2 \mathrm{mg} \mathrm{mAb} / \mathrm{kg}$ body weight) or isotype control antibody by intraperitoneal injection $24 \mathrm{~h}$ before intranasal infection with a lethal dose of MERS-CoV. Data points represent mean body weight relative to the initial weight $\pm S D, n=5$ mice. Source data are provided as a Source Data file. c Prophylactic efficacy of 28D9 against SARS-CoV infection. Five 16-week-old Balb-C mice were mock-infected or administered with the 50 or $200 \mu \mathrm{g}$ of $28 \mathrm{D} 9$ or isotype control antibody via intraperitoneal injection $24 \mathrm{~h}$ before intranasal infection with a lethal dose of mouse-adapted SARS-CoV. Survival rates (left) and weight loss (right, expressed as a percentage of the initial weight) were monitored daily until 13 days post-inoculation. Data points represent mean body weight relative to the initial weight $\pm S D, n=5$ mice. Source data are provided as a Source Data file.

polymorphisms were observed in the stem-helix epitope in all available 160 spike protein sequences of human MERS-CoV isolates present in the VIPR database (https://www.viprbrc.org/), suggesting broad-neutralization capacity of both antibodies against MERS-CoV isolates. The epitope containing region is engaged in extensive conformational rearrangements in the S2 subunit, that drive membrane fusion. Elucidation of pre- and post-fusion spike structures reveals that dissociation of the three epitope-containing stem-helices in the prefusion spike trimer is required for the formation of the highly stable postfusion structure $31,52,59,60$. Though the precise mechanism of neutralization by these two antibodies remains to be defined, at least two mechanisms of neutralization are conceivable: the binding of antibodies may destabilize the bundle of stem helices in the prefusion spike protein initiating premature spike activation. Alternatively, antibody binding may obstruct six-helix bundle 
formation of the spike protein during fusion ${ }^{61}$. Intriguingly, despite having different conformations resulting from the structural rearrangement during fusion, the epitope in pre- and post-fusion spike conformation could still be bound by both antibodies, suggesting either epitope recognition of the structurally conserved part of the epitope in the two spike conformations, or through an 'induced fit' mechanism at the binding interface ${ }^{62}$.

Neutralization of authentic human-infecting coronaviruses, other than MERS-CoV, was not observed by these two antibodies, although weak cross-neutralizing capacity against pseudotyped viruses was seen for 28D9. This monoclonal antibody displayed high ELISA-reactivity to the spike proteins of $\mathrm{HCoV}-\mathrm{OC} 43$, SARS-CoV, SARS-CoV-2. The lack of robust cross-neutralization by $28 \mathrm{D} 9$ may result from the $\sim 5$ - to 10 -fold higher equilibrium dissociation constants towards these spike proteins, relative to MERS-S, inferred by subtle differences in epitope composition. To increase neutralization breadth and potency of the crossreactive antibodies, antibody affinity maturation could be explored using a directed evolution approach following antibody mutagenesis ${ }^{63}$. In addition, structural insight into the 28D9 antibody binding mode to the stem helix epitope of targeted $\mathrm{CoV}$ spike proteins may guide antibody affinity improvement by structure-based design to increase breadth and potency of neutralization.

The conserved stem helix epitope appears to be highly immunogenic. This is demonstrated by the independent isolation of three cross-reactive antibodies $(28 \mathrm{D} 9,1.6 \mathrm{C} 7$ and $18 \mathrm{H} 2)$ all targeting the stem-helix epitope from three independent mouse immunization experiments. In addition, antibodies towards this epitope are also elicited during natural infection, as demonstrated by the spike protein peptide microarray analysis using MERS$\mathrm{CoV}$-positive human and dromedary camel sera. Other studies also highlight the immunogenicity of this epitope region during natural infection. A recent preprint study reports a cross-reactive and neutralizing $\mathrm{mAb}$ CC40.8 isolated from a COVID-19 patient that targets the S2 subunit of the S protein. Using negative stain electron microscopy, the antibody was shown to bind at a membrane-proximal position in the membrane fusion subunit S2, similar to 28D964. Moreover, recent spike protein peptide microarray analyses by Li et al. ${ }^{65}$ revealed that the SARS-CoV-2 peptide $\mathrm{F}^{1148} \mathrm{KEELDKYFKNH}^{1159}$ encompassing the 28D9/1.6C7 targeted stem helix epitope EELDKYFK was recognized by serum antibodies in $\sim 90 \%$ of COVID-19 patients, whereas Ladner et al. ${ }^{66}$ also identifies the stem-helix epitope region-with $\mathrm{F}^{1148} \mathrm{KEELDKYF}^{1156}$ as the minimal reactive SARS2-S peptide sequence-as the most widely-recognized SARS-CoV-2 linear epitope target in convalescent donors. It is tempting to speculate that the exceptionally high seroprevalence of stem-epitope targeting antibodies in COVID-19 patients is due to boosting of preexisting immune response towards this conserved epitope resulting from earlier encounters with betacoronaviruses such as HCoV-OC43 and HCoV-HKU1 that are endemic in humans ${ }^{21}$. To what extent cross-reactive antibodies in humans towards the stem-helix or other conserved spike epitopes exist and play a role in cross-protection or enhanced disease requires further investigation.

Together, the isolated cross-reactive antibodies define a conserved, immunogenic and vulnerabe site on the coronavirus spike protein. The discovery of eptiopes on viral glycoproteins targeted by cross-reactive neutralizing antibodies has fuelled the design of broad range therapeutics and vaccines for other RNA viruses that display antigenic variation or show zoonotic potential, such as HIV-1 and influenza viruses ${ }^{13,15,67-69}$. We made a first step into the identification of conserved sites among antigenically highly divergent coronavirus spike proteins. Unveiling additional conserved sites on the coronavirus spike protein may enable the new generation of broadly protective (epitope-focused) vaccines and therapeutic cocktails, that can mitigate the potential risk of antigenic drift upon continuous circulation of coronaviruses in the population, as well as the looming threat of novel coronavirus emergence in humans.

\section{Methods}

Expression and purification of coronavirus spike proteins. Coronavirus spike ectodomains of MERS-CoV (residues 19-1262; strain EMC; GenBank accession number (GB): YP_009047204.1), SARS-CoV (residues 15-1182; strain Urbani; GB: AY278741.1), MHV (residues 15-1231; strain A59; UniProt accession number: P11224) and the S2 ectodomain of MHV (residues 718-1252; strain A59; UniProt accession number: P11224) fused with a C-terminal T4/GCN4 trimerization motif, a thrombin cleavage site and a Strep-tag purification tag were cloned in-frame into $\mathrm{pMT} \backslash \mathrm{Bip} \backslash \mathrm{V} 5 \backslash$ His expression vector. The furin cleavage site at the S1/S2 junction was mutated to prevent cleavage by furin at this position. Spike ectodomains were stably produced in Drosophila S2 cell line, as previously described ${ }^{28}$. Spike ectodomains of SARS-CoV-2 (residues 1-1,213; strain Wuhan-Hu-1; GenBank: QHD43416.1), HCoV-OC43 (residues 15-1263; strain Paris; UniProtKB: Q696P8) and HCoV-HKU1 (residues 14-1266; strain Caen1; GenBank: HM034837) were expressed transiently in HEK-293T (ATCC ${ }^{\circledR}$ CRL-11268 ${ }^{\text {su}}$ ) cells with a C-terminal trimerization motif and Strep-tag using the pCAGGS expression plasmid. The genes encoding MERS-S2 ectodomain (residues 752-1262; strain EMC; GB: YP_009047204.1) were cloned in-frame between the HBM secretion signal peptide and a triple Strep-tag for purification in the pFastBac transfer vector. Generation of bacmid DNA and recombinant baculovirus was performed according to protocols from the Bac-to-Bac system (Invitrogen). All secreted proteins were purified from culture supernatant using streptactin beads (IBA) following the manufacturer's protocol. All variants were generated using Q $5^{\circledR}$ High-fidelity DNA polymerase (NEB)-based site-directed mutagenesis.

Generation of cross-reactive H2L2 mAbs. Six H2L2 mice were sequentially immunized in two weeks intervals with purified spike ectodomains of different CoVs in the following order ${ }^{34}$ : HCoV-OC43, SARS-CoV, MERS-CoV, HCoVOC43, SARS-CoV and MERS-CoV. Antigens were injected at 20-25 $\mu \mathrm{g} /$ mouse using Stimune Adjuvant (Prionics) freshly prepared according to the manufacturer's instruction for the first injection, while boosting was done using Ribi (Sigma) adjuvant. Injections were done subcutaneously into the left and right groin each $(50 \mu \mathrm{l})$ and $100 \mu \mathrm{l}$ intraperitoneally. Four days after the last injection, spleen and lymph nodes were harvested, and hybridomas made by standard method using SP 2/0 myeloma cell line (ATCC \#CRL-1581) as a fusion partner. Hybridomas were screened in an antigen-specific ELISA and positives selected for further development, subcloned and produced on a small scale ( $100 \mathrm{ml}$ of medium). For this purpose, hybridomas were cultured in serum- and protein-free medium for hybridoma culturing (PFHM-II (1X), Gibco) with the addition of non-essential amino acids (100X NEAA, Biowhittaker Lonza, cat. no. BE13-114E). H2L2 antibodies were purified from hybridoma culture supernatants using Protein-G affinity chromatography. Purified antibodies were stored at $4{ }^{\circ} \mathrm{C}$ for further use. DNA immunizations of H2L2 mice were done in two weeks intervals with spike protein expression plasmids in the following order: pCAGGS-OC43-S, pCAGGS-SARS-S and pCAGGS-MERS-S. During the procedure that involved shaving of the lower back, intradermal DNA injection $(40-50 \mu \mathrm{g}$ of DNA per mouse in $20-30 \mu \mathrm{l}$ volume) and electroporation, mice were anesthetized. Electroporation was done according to instructions of the manufacturer of the electroporation apparatus (Agile plus ID in vivo delivery system, BTX). In short, immediately after the DNA injection, mice were subjected to 10 electro pulses using $2 \times 6$ array needle surrounding the bleb (small blister under the skin) formed after the injection. Anaesthesia was given following standard operation procedures of the facility. Blood samples will be taken after the 4th DNA injectiion/electroporation. Five mice that developed satisfactory ELISA titres for all the three antigens after DNA priming were additionally injected subcutaneously in two weeks intervals with $25 \mu \mathrm{g}$ of each of the soluble trimeric spike proteins with Ribi adjuvant. 3-5 days after the last injection, mice were sacrificed and spleens and lymph nodes used to make a single-cell suspension for the fusion experiment.

All H2L2 mice were housed in an SPF (specific pathogen free) facility in individually ventilated cages (IVC), with cage enrichment, light switched on at 7:00 and switched off at 19:00 and with humidity at around 40\%. Both female and male H2L2 mice were equally used and were housed in separate cages. Animal studies were done under the animal permit AVD101002016512, under work protocol 16 512-22 called "heterologous prime-boost approach", approved by the CCD (central committee for animal experiments).

Production of recombinant human monoclonal antibodies. Production of recombinant human antibodies using HEK-293T was described previously ${ }^{43}$ Briefly, the variable heavy (VH) and light (VL) chain sequences were amplified from cDNA and separately cloned into the expression plasmids with human IgG1 heavy chain and kappa chain constant regions, respectively (Invivogen). Both plasmids contain the interleukin-2 signal sequence to enable the efficient secretion 
of recombinant antibodies. Recombinant human antibodies were expressed in HEK-293T cells following transient transfection with pairs of the IgG1 heavy and light chain expression plasmids according to protocols from Invivogen. Recombinant antibodies were purified using Protein A sepharose (IBA) according to the manufacturer's instruction. $\mathrm{mAb} 1.6 \mathrm{C} 7$ and $7.7 \mathrm{G} 6$ used in the animal experiments were produced in Chinese hamster ovary (CHOK1-derived, ECACC 85051005) cells as previously described ${ }^{70}$. The VH and VL sequences were synthesized by GeneArt, ThermoFisher and cloned into OriP-containing expression vectors, suitable for IgG1 production ${ }^{71}$. A $20 \mathrm{~L}$ cell culture in a disposable rocking bioreactor was subsequently transiently transfected with the heavy and light chain expression vectors. The clarified harvest supernatant was purified using Protein A-based chromatography ${ }^{72}$.

ELISA analysis of antibody binding to CoV spike antigens. Purified coronavirus spike ectodomains were coated onto 96-well NUNC Maxisorp plates (Thermo Scientific) at equimolar amount at room temperature (RT) for $3 \mathrm{~h}$ followed by three washing steps with Phosphate Saline Buffer (PBS) containing 0.05\% Tween-20. Plates were blocked with 5\% milk (Protifar, Nutricia) in PBS with $0.1 \%$ Tween-20 at $4{ }^{\circ} \mathrm{C}$ overnight. Antibodies were allowed to bind to the plates at fourfold serial dilutions, starting at $10 \mu \mathrm{g} / \mathrm{ml}$ diluted in PBS containing 3\% BSA and $0.1 \%$ Tween20, at RT for $1 \mathrm{~h}$. Antibody binding to the spike proteins was determined using a 1:2000 diluted HRP-conjugated goat anti-human IgG (ITK Southern Biotech) for $1 \mathrm{~h}$ at RT and tetramethylbenzidine substrate (BioFX). Readout for binding was done at $450 \mathrm{~nm}\left(\mathrm{OD}_{450}\right)$ using the ELISA plate reader (EL-808, Biotek). Half-maximum effective concentration $\left(\mathrm{EC}_{50}\right.$ ) binding values were calculated by 4-parameter logistic regression on the binding curves using GraphPad Prism version 7.04. To determine whether antibodies recognize a linear or a discontinuous epitope, NUNC Maxisorp plates were coated with $100 \mathrm{ng} /$ well of MERS-S ectodomain at RT for $3 \mathrm{~h}$. Antigens were treated with or without $50 \mu \mathrm{l}$ of denaturing buffer (200 mM DTT and $4 \%$ SDS in PBS) at $37^{\circ} \mathrm{C}$ for $1 \mathrm{~h}$. After three times washing steps with PBST (PBS with $0.05 \%$ Tween-20), plates were blocked by blocking buffer ( $5 \%$ milk in PBS with $0.1 \%$ Tween-20) at $4{ }^{\circ} \mathrm{C}$ overnight. Fourfold serially diluted primary antibodies were added to the plates and incubate at RT for $1 \mathrm{~h}$. Plates were washed three times and antibody binding to the spike proteins was analysed as described above.

Immunofluorescence microscopy. Antibody binding to cells expressing spike proteins of MERS, SARS-CoV, SARS-CoV-2, MHV, HCoV-OC43 and HCoVHKU1 was measured by immunofluoresence microscopy. HEK-293T cells seeded on glass slides were transfected with plasmids encoding MERS-S, SARS-S, SARS2S, MHV-S, HCoV-OC43-S or HCoV-HKU1-S C-terminally fused to the green fluorescence protein (GFP) using Lipofectamine 2000 (Invitrogen). Two days post transfection, cells were fixed by incubation with $2 \%$ paraformaldehyde in PBS for $20 \mathrm{~min}$ at RT before $0.1 \%$ Triton- 100 permeabilization and stained for nuclei with 4,6-diamidino-2-phenylindole (DAPI). Cells were subsequently incubated with mAbs at a concentration of $10 \mu \mathrm{g} / \mathrm{ml}$ for $1 \mathrm{~h}$ at RT, followed by incubation with 1:200 diluted Alexa Fluor 594 conjugated goat anti-human IgG antibody (Invitrogen, Thermo Fisher Scientific) for $45 \mathrm{~min}$ at RT. The fluorescence images were recorded using a Leica SpeII confocal microscope.

Flow cytometry. HEK-293T cells were seeded with a density of $3 \times 10^{6}$ cells per T25 tissue culture flask. After reaching $70-80 \%$ confluency, cells were transfected with the pCAGGS expression plasmids encoding full-length spikes of MERS-CoV, SARS-CoV, SARS-CoV-2, MHV, HCoV-OC43 and HCoV-HKU1 C-terminally extended with GFP. Two days post transfection, cells were harvested by cell dissociation solution (Sigma-aldrich, Merck KGaA; cat. no. C5914). Single-cell suspensions in FACS buffer (2\% foetal bovine serum (FBS), $5 \mathrm{mM}$ EDTA and $0.02 \%$ $\mathrm{NaN}_{3}$ in PBS) were centrifuged at $400 \times g$ for $10 \mathrm{~min}$. Cells were then treated with/ without $0.1 \%$ Triton- 100 after fixation in $3.7 \%$ paraformaldehyde. After a washing step in PBS, cells were blocked using 10\% Normal Goat Serum (Gibco, Thermo Fisher Scientific, the Netherlands) diluted in PBS for $45 \mathrm{~min}$ at RT. Staining of spike proteins was performed by incubation of the cells with primary antibody $(10 \mu \mathrm{g} / \mathrm{ml})$ for $1 \mathrm{~h}$ at RT. They were then incubated with 1:200 diluted Alexa Fluor 594 conjugated goat anti-human IgG secondary antibody (Invitrogen, Thermo Fisher Scientific) for $45 \mathrm{~min}$ at RT and subjected to flow cytometric analysis with a CytoFLEX flow cytometer (Beckman). The results were analysed by FlowJo (version 10) and percentage of $\mathrm{GFP}^{+}$Alexa Fluor $594^{+}$cells over $\mathrm{GFP}^{+}$cells were calculated.

Antibody binding kinetics and affinity measurement. The measurement of binding kinetics and affinity of antibodies to $\mathrm{CoV}$ spike ectodomains was performed using biolayer interferometry (Octet RED384 machine) as described before ${ }^{43}$. Briefly, fully human antibodies with optimal concentration $(44 \mathrm{nM})$ which showed the desired loading curve characteristics and high signal in the association step were loaded onto Protein A biosensors for $10 \mathrm{~min}$. The binding of CoV spikes was performed by incubating the biosensor with various concentrations of recombinant spike ectodomain (1600-800-400-200-100-50-25-12.5-6.25 nM) for $10 \mathrm{~min}$ followed by the dissociation step which was run long enough $(60 \mathrm{~min})$ to observe the decrease of the binding response. The affinity constant $K_{D}$ was calculated using 1:1 Langmuir binding model on Fortebio Data Analysis 7.0 software.

Pseudotyped virus neutralization assay. The production of coronavirus spike pseudotyped VSV virus and the neutralization assay was performed as described previously 43,73 . In brief, HEK-293T cells at $70-80 \%$ confluency were transfected with the pCAGGS expression vectors encoding full-length MERS-S, SARS-S, SARS2-S or OC43-S with a C-terminal cytoplasmic tail truncation to increase cell surface expression levels. In the case of OC43-S, cells were co-transfected with pCAGGS vector encoding the Fc-tagged bovine coronavirus hemagglutinin esterase $(\mathrm{HE}-\mathrm{Fc})$ protein at molar ratios of 8:1 (S:HE-Fc). Forty-eight hours post transfection, cells were infected with VSV-G pseudotyped VSV $\Delta$ G bearing the firefly (Photinus pyralis) luciferase reporter gene at a MOI of 1 . Twenty-four hours later, the supernatant was harvested and filtered through $0.45 \mu \mathrm{m}$ membrane. Pseudotyped VSV virus were titrated on monolayer African green monkey kidney VeroCCL81 cells (MERS-S pseudotyped VSV; ATCC ${ }^{\circledR}$ CCL-81 ${ }^{\text {m) }}$ ), VeroE6 cells (SARS-S and SARS2-S pseudotyped VSV; ATCC ${ }^{\oplus}$ CRL-1586 ${ }^{\mathrm{mt}}$ ) or on HRT-18 cells (OC43-S pseudotyped VSV; ATCC ${ }^{\circledast}$ CCL-244 ${ }^{\mathrm{m}}$ ). In the virus neutralization assay, serially diluted $\mathrm{mAbs}$ were pre-incubated with an equal volume of the virus at RT for $1 \mathrm{~h}$, and then inoculated on Vero/HRT-18 cells, and further incubated at $37^{\circ} \mathrm{C}$. After $20 \mathrm{~h}$, cells were washed once with PBS and lysed with cell lysis buffer (Promega). The expression of firefly luciferase was measured on a Berthold Centro LB 960 plate luminometer using D-luciferin as a substrate (Promega). The percentage of infectivity was calculated as the ratio of luciferase readout in the presence of mAbs normalized to luciferase readout in the absence of $\mathrm{mAb}$. The halfmaximal inhibitory concentrations $\left(\mathrm{IC}_{50}\right)$ were determined using 4-parameter logistic regression (GraphPad Prism v7.0).

Authentic virus neutralization assay. Neutralization of authentic MERS-CoV, SARS-CoV and SARS-CoV-2 was performed using a plaque reduction neutralization test (PRNT) as described earlier, with some modifications ${ }^{74,75}$. In brief, mAbs were serially diluted and mixed with MERS-CoV, SARS-CoV or SARS-CoV2 for $1 \mathrm{~h}$. The mixture was then added to Huh-7 cells (MERS-CoV; JCRB Cell Bank, JCRB0403) or VeroE6 cells (SARS-CoV and SARS-CoV-2) and incubated for $1 \mathrm{~h}$, after which the cells were washed and further incubated in a medium for $8 \mathrm{~h}$ Subsequently, the cells were washed, fixed, permeabilized and the infection was detected using immunofluorescent staining using antibodies specific for the viruses used. The signal was developed using a precipitate forming TMB substrate (True Blue, KPL) and the number of infected cells per well were counted using the ImmunoSpot ${ }^{\circledR}$ Image analyzer (CTL Europe GmbH). The half-maximal inhibitory concentrations $\left(\mathrm{IC}_{50}\right)$ were determined using 4-parameter logistic regression (GraphPad Prism version 8).

Receptor-binding inhibition assay. The DPP4 receptor-binding inhibition assay was performed as described previously ${ }^{43}$. Recombinant soluble DPP4 was coated on NUNC Maxisorp plates (Thermo Scientific) at $100 \mathrm{ng} / \mathrm{well}$ at RT for $3 \mathrm{~h}$. Plates were washed three times with PBS containing $0.05 \%$ Tween- 20 and blocked with $5 \%$ milk (Protifar, Nutricia) in PBS containing $0.1 \%$ Tween- 20 at $4{ }^{\circ} \mathrm{C}$ overnight. Recombinant MERS-CoV S ectodomain and serially diluted mAbs were mixed and incubated for $1 \mathrm{~h}$ at RT. The mixture was added to the plate for $1 \mathrm{~h}$ at RT, after which plates were washed three times. The binding of MERS-CoV S ectodomain to DPP4 was detected using 1:1000 diluted HRP-conjugated anti-StrepMAb (IBA) that recognizes the Strep-tag affinity tag on the MERS-CoV S ectodomain. Detection of HRP activity was performed as described above (ELISA section).

Fusion inhibition assay. Fusion inhibition assay was perfomed as described ${ }^{43}$, with some adaptations. Huh-7 cells were seeded one day before reaching a confluency of $70-80 \%$. Cells were transfected with pCAGGS expression plasmid encoding full-length MERS-S C-terminally fused with a GFP-tag using Lipofectamine 2000. The furin cleavage site $R^{747} \mathrm{SVR}^{751}$ at $\mathrm{S} 1 / \mathrm{S} 2$ junction was mutated to $K S V R$ to avoid the cleavage by endogenous proteases. At $48 \mathrm{~h}$ post transfection, cells were pre-treated with DMEM only or DMEM with $20 \mu \mathrm{g} / \mathrm{ml} \mathrm{mAbs} \mathrm{for} 1 \mathrm{~h}$ and subsequently treated with DMEM with $20 \mu \mathrm{g} / \mathrm{ml}$ of exogenous trypsin to activate the spike fusion function at $37^{\circ} \mathrm{C}$ for $2 \mathrm{~h}$. Cells were fixed with $3.7 \%$ paraformaldehyde after observation of the syncytia formation. 4,6-diamidino-2-phenylindole (DAPI) was used to stain the nuclei. The expression of MERS-S was confirmed based on the GFP signal, and the cell-cell fusion was monitored by large GFP-fluorescent muti-nucleated syncytia. The fluorescence images were recorded using the EVOS FL fluorescence microscope (Thermo Fisher Scientific, the Netherlands).

Antibody binding competition assay. Antibody binding competition was performed using biolayer interferometry (Octet Green; ForteBio), as described previously ${ }^{43}$. In brief, MERS-CoV spike antigen $50 \mu \mathrm{g} / \mathrm{ml}$ was immobilized onto the anti-strep mAb-coated protein A biosensor. After a brief washing step, the biosensor tips were immersed into a well containing primary $\mathrm{mAb}$ at a concentration of $50 \mu \mathrm{g} / \mathrm{ml}$ for $15 \mathrm{~min}$ and subsequently into a well containing the competing $\mathrm{mAb}$ (secondary $\mathrm{mAb}$ ) at a concentration of $50 \mu \mathrm{g} / \mathrm{ml}$ for $15 \mathrm{~min}$. A 5-min washing step in PBS was included in between steps. 
Spike protein peptide microarray analysis. Spike protein peptide microarray analysis to map linear epitopes of antibodies in convalescent sera was performed by PEPSCAN (Lelystad, The Netherlands). Overlapping peptides that cover the entire MERS-CoV spike ectodomain (residues 1-1296) were synthesized with an offset of one or two residues. The order of these peptides was randomized, when synthesized on mini-cards. ELISA reactivity of five human (H1-H5) and four dromedary camel (D1-D4) MERS-positive sera was assessed, as well as a MERS-negative serum from human (H-CTRL) or camel (D-CTRL). The binding of antibody to each of the synthesized peptides was tested in a PEPSCAN-based ELISA. The peptide arrays were incubated with the primary antibody solution (overnight at $4^{\circ} \mathrm{C}$ ). After washing, the peptide arrays were incubated with a $1 / 1000$ dilution of an appropriate antibody peroxidase conjugate (goat anti-human HRP conjugate, Southern Biotech, cat. no.: 2010-05 or goat anti-lama HRP conjugate, Abcore, cat. no. AC15-0354) for $1 \mathrm{~h}$ at $25^{\circ} \mathrm{C}$. After washing, the peroxidase substrate $2,2^{\prime}$-azinodi-3-ethylbenzthiazoline sulfonate (ABTS) and $20 \mu \mathrm{l} / \mathrm{ml}$ of $3 \% \mathrm{H}_{2} \mathrm{O}_{2}$ was added. After $1 \mathrm{~h}$, the colour development was measured and quantified with a chargecoupled device $(C C D)$ - camera and an image processing system. The values obtained from the CCD camera range from 0 to $3000 \mathrm{mAU}$. Samples were scaled per serum sample using a cut-off of twice the mean absorbance obtained for each serum. The use of human materials was approved by the Erasmus MC medical ethical committee (MEC approval: 2014-414) and written informed consent was obtained from participants.

To map the epitopes of the monoclonal antibodies, 30-amino acid long peptides (with 15-a.a. overlap) were synthesized (Genscript) covering the conserved Cterminal part of the MERS-S2 ectodomain (residues 869-1288). $100 \mathrm{ng} / \mathrm{well}$ of each peptide was coated onto 96-well NUNC Maxisorb plate at $4{ }^{\circ} \mathrm{C}$ overnight. Followed by three washing steps with PBST (PBS with $0.05 \%$ Tween-20), plates were blocked with 5\% milk (Protifar, Nutricia) in PBS with $0.1 \%$ Tween-20 at RT for $3 \mathrm{~h}$. Antibodies were allowed to bind to the plates at 4 -fold serial dilutions, starting at $10 \mu \mathrm{g} / \mathrm{ml}$, at RT for $1 \mathrm{~h}$. Antibody binding to the peptides was determined using a goat anti-human IgG HRP conjugate (ITK Southern Biotech) for $1 \mathrm{~h}$ at RT and tetramethylbenzidine substrate (BioFX). Readout for binding was done as described in the ELISA section. To identify critical residues for $\mathrm{mAb}$ binding, a single alanine mutation was introduced on the 15-mer spike peptide fragment that comprises the linear epitope. Reactivity of antibodies with peptides with a single alanine substitution was measured by ELISA according to the method described above.

Passive immunization and protection tests of mice. In vivo prophylactic and therapeutic efficacy of $1.6 \mathrm{C} 7$ against MERS-CoV infection was evaluated in the transgenic mouse model K18 TghDpp4 expressing the receptor for the human MERS-CoV 53 . Groups of 8 mice, 20 -30-weeks old, were given $50 \mu \mathrm{g}$ of $1.6 \mathrm{C} 7$ (equivalent to $1.8 \mathrm{mg}$ of the antibody per $\mathrm{kg}$ ) by intraperitoneal injection, $24 \mathrm{~h}$ before or after intranasal infection with a lethal dose of MERS-CoV (EMC isolate; $5 \times 10^{3} \mathrm{PFU} /$ mouse). The potent neutralizing anti-MERS-S1 control antibody $7.7 \mathrm{G} 6^{43}$ and an isotype-matched negative control $\mathrm{mAb}$ were taken along as controls. In a second experiment, the prophylactic efficacy of mAb 28D9 has tested against MERS-CoV and SARS-CoV infection in the K18 TghDpp4 mouse model. Groups of $16-20$-week-old mice $(n=5)$, were given 50 or $200 \mu \mathrm{g} 28 \mathrm{D} 9$ or isotype control antibody (equivalent to 1.8 or $7.2 \mathrm{mg}$ of the antibody per $\mathrm{kg}$, respectively) by intraperitoneal injection, $24 \mathrm{~h}$ before intranasal infection with a lethal dose of MERS-CoV (EMC isolate; $5 \times 10^{3} \mathrm{PFU} /$ mouse) or mouse-adapted SARS-CoV (strain SARS-CoV-MA15-WT-M2; $1 \times 10^{5} \mathrm{PFU} /$ mouse). All animals were housed in a self-contained ventilated rack (Allentown, NJ), with the light switched on at 7:30 and switched off at $19: 30$. The ambient temperature is $19.5-22^{\circ} \mathrm{C}$ and with humidity at $35-40 \%$. Animal protection studies were done under the animal permit PROEX-199/19, approved by the Community of Madrid (Spain), and performed in biosafety level 3 facilities at CISA-INIA (Madrid).

MERS-CoV titers and lung histopathology were tested as described earlier ${ }^{74}$. To analyze MERS-CoV titers, one fourth of the right lung was homogenized using a MACS homogenizer (Miltenyi Biotec) according to the manufacturer's protocols. Virus titrations were performed on Huh-7 cells following standard procedures. In brief, cells were overlaid with DMEM containing $0.6 \%$ low-melting agarose and $2 \%$ FBS, fixed with $10 \%$ formaldehyde and stained with $0.1 \%$ crystal violet at $72 \mathrm{~h}$ post infection. The left lung of infected mice was fixed in $10 \%$ zinc formalin for $24 \mathrm{~h}$ at $4{ }^{\circ} \mathrm{C}$ and paraffin embedded for lung histopathological examination. Serial longitudinal $5-\mu \mathrm{m}$ sections of formaling were stained with hematoxylin and eosin (H\&E) and subjected to histopathological examination with a ZEISS Axiophot fluorescence microscope. Samples were obtained using a systematic uniform random procedure, consisting in serial parallel slices made at a constant thickness interval of $50 \mu \mathrm{m}$.

Reporting summary. Further information on research design is available in the Nature Research Reporting Summary linked to this article.

\section{Data availability}

Data underlying Figs. 1a, 2a-d, 3a-d, 4a, 4c-d, 6b-c, 7a-c, Supplementary Table 1, Supplementary Figs 2, 3, 5, 6, 7, 8a-e, 9, 10a-b, 11, 12 and 13a are provided as Source Data files in a publicly accessible repository (https://figshare.com/s/ 39254c60572bb7b8bc0d). PDB files of SARS2 spike protein (PDB ID: 6XR8 and 6XRA) were downloaded from NCBI database (https://www.ncbi.nlm.nih.gov/). Spike protein sequences used in this study were downloaded from NCBI database (https://www.ncbi. nlm.nih.gov/) (See supplementary figure 11 for the accession numbers). Sequences of the monoclonal antibodies characterized here are available from GenBank under the following accession numbers: MW579767, MW579768, MW579769, MW579770, MW579771 and MW579772. All other data are available from the corresponding author upon reasonable requests. Source data are provided with this paper.

Received: 20 October 2020; Accepted: 15 February 2021; Published online: 17 March 2021

\section{References}

1. Zhou, P. et al. A pneumonia outbreak associated with a new coronavirus of probable bat origin. Nature 579, 270-273 (2020).

2. Gorbalenya, A. et al. Coronaviridae Study Group of the International Committee on Taxonomy of Viruses. The species severe acute respiratory syndrome-related coronavirus: classifying $2019-\mathrm{nCoV}$ and naming it SARSCoV-2. Nat. Microbiol. 2020, 03-04 (2020).

3. Peiris, J., Guan, Y. \& Yuen, K. Severe acute respiratory syndrome. Nat. Med 10, S88-S97 (2004)

4. World Health Organization. MERS situation update, January 2020. http:// www.emro.who.int/health-topics/mers-cov/mersoutbreaks.html (2020).

5. Gaunt, E. R., Hardie, A., Claas, E. C., Simmonds, P. \& Templeton, K. E. Epidemiology and clinical presentations of the four human coronaviruses 229E, HKU1, NL63, and OC43 detected over 3 years using a novel multiplex real-time PCR method. J. Clin. Microbiol. 48, 2940-2947 (2010).

6. Lim, Y. X., Ng, Y. L., Tam, J. P. \& Liu, D. X. Human coronaviruses: a review of virus-host interactions. Diseases 4, 26 (2016).

7. Walsh, E. E., Shin, J. H. \& Falsey, A. R. Clinical impact of human coronaviruses $229 \mathrm{E}$ and OC43 infection in diverse adult populations. J. Infect. Dis. 208, 1634-1642 (2013).

8. Morfopoulou, S. et al. Human coronavirus OC43 associated with fatal encephalitis. N. Engl. J. Med. 375, 497-498 (2016).

9. Su, S. et al. Epidemiology, genetic recombination, and pathogenesis of coronaviruses. Trends Microbiol. 24, 490-502 (2016).

10. Chan, J. F., To, K. K., Tse, H., Jin, D. \& Yuen, K. Interspecies transmission and emergence of novel viruses: lessons from bats and birds. Trends Microbiol. 21, 544-555 (2013).

11. Hu, B., Ge, X., Wang, L. \& Shi, Z. Bat origin of human coronaviruses. Virol. J. 12, 1-10 (2015)

12. Burton, D. R. \& Walker, L. M. Rational vaccine design in the time of COVID19. Cell Host Micro. 27, 695-698 (2020).

13. Burton, D. R., Poignard, P., Stanfield, R. L. \& Wilson, I. A. Broadly neutralizing antibodies present new prospects to counter highly antigenically diverse viruses. Science 337, 183-186 (2012).

14. Pappas, L. et al. Rapid development of broadly influenza neutralizing antibodies through redundant mutations. Nature 516, 418-422 (2014).

15. Chen, Y. et al. Influenza infection in humans induces broadly cross-reactive and protective neuraminidase-reactive antibodies. Cell 173, 417-429.e10 (2018).

16. Flyak, A. I. et al. Cross-reactive and potent neutralizing antibody responses in human survivors of natural ebolavirus infection. Cell 164, 392-405 (2016).

17. Wec, A. Z. et al. Antibodies from a human survivor define sites of vulnerability for broad protection against ebolaviruses. Cell 169, 878-890.e15 (2017).

18. Gilchuk, P. et al. Multifunctional pan-ebolavirus antibody recognizes a site of broad vulnerability on the ebolavirus glycoprotein. Immunity 49, 363-374 (2018). e10.

19. Chan, K. et al. Cross-reactive antibodies in convalescent SARS patients' sera against the emerging novel human coronavirus EMC (2012) by both immunofluorescent and neutralizing antibody tests. J. Infect. 67, 130-140 (2013).

20. Barnes, C. O. et al. Structures of human antibodies bound to SARS-CoV-2 spike reveal common epitopes and recurrent features of antibodies. Cell 184, 828-842 (2020).

21. Wec, A. Z. et al. Broad neutralization of SARS-related viruses by human monoclonal antibodies. Science 369, 731-736 (2020).

22. Kirchdoerfer, R. N. et al. Stabilized coronavirus spikes are resistant to conformational changes induced by receptor recognition or proteolysis. Sci. Rep. 8, 1-11 (2018)

23. Li, Z. et al. The human coronavirus HCoV-229E S-protein structure and receptor binding. Elife 8, e51230 (2019).

24. Park, Y. et al. Structures of MERS-CoV spike glycoprotein in complex with sialoside attachment receptors. Nat. Struct. Mol. Biol. 26, 1151-1157 (2019). 
25. Shang, J. et al. Cryo-EM structure of infectious bronchitis coronavirus spike protein reveals structural and functional evolution of coronavirus spike proteins. PLoS Pathog. 14, e1007009 (2018).

26. Shang, J. et al. Cryo-Electron Microscopy Structure of Porcine Deltacoronavirus Spike Protein in the Prefusion State. J. Virol. https://doi.org/ 10.1128/JVI.01556-17 (2018).

27. Tortorici, M. A. et al. Structural basis for human coronavirus attachment to sialic acid receptors. Nat. Struct. Mol. Biol. 26, 481-489 (2019).

28. Walls, A. C. et al. Cryo-electron microscopy structure of a coronavirus spike glycoprotein trimer. Nature 531, 114-117 (2016).

29. Walls, A. C. et al. Unexpected receptor functional mimicry elucidates activation of coronavirus fusion. Cell 176, 1026-1039.e15 (2019).

30. Yuan, Y. et al. Cryo-EM structures of MERS-CoV and SARS-CoV spike glycoproteins reveal the dynamic receptor binding domains. Nat. Commun. 8 , 1-9 (2017).

31. Cai, Y. et al. Distinct conformational states of SARS-CoV-2 spike protein. Science 369, 1586-1592 (2020).

32. Wrapp, D. et al. Cryo-EM structure of the 2019-nCoV spike in the prefusion conformation. Science 367, 1260-1263 (2020).

33. Menachery, V. D. et al. A SARS-like cluster of circulating bat coronaviruses shows potential for human emergence. Nat. Med. 21, 1508-1513 (2015).

34. Wang, C. et al. A human monoclonal antibody blocking SARS-CoV-2 infection. Nat. Commun. 11, 1-6 (2020).

35. Zhou, D. et al. Structural basis for the neutralization of SARS-CoV-2 by an antibody from a convalescent patient. Nat. Struct. Mol. Biol. 27, 950-958 (2020).

36. Brouwer, P. et al. Potent neutralizing antibodies from COVID-19 patients define multiple targets of vulnerability. Science 369, 643-650 (2020).

37. Lv, H. et al. Cross-reactive antibody response between SARS-CoV-2 and SARS-CoV infections. Cell Rep. 31, 107725 (2020).

38. Pinto, D. et al. Cross-neutralization of SARS-CoV-2 by a human monoclonal SARS-CoV antibody. Nature 583, 290-295 (2020).

39. Lv, Z. et al. Structural basis for neutralization of SARS-CoV-2 and SARS-CoV by a potent therapeutic antibody. Science 369, 1505-1509 (2020).

40. Rogers, T. F. et al. Isolation of potent SARS-CoV-2 neutralizing antibodies and protection from disease in a small animal model. Science 369, 956-963 (2020).

41. Haagmans, B. L. et al. SARS-CoV-2 neutralizing human antibodies protect against lower respiratory tract disease in a hamster model. Preprint at https:// doi.org/10.1101/2020.08.24.264630 (2020).

42. Walls, A. C. et al. Structure, function, and antigenicity of the SARS-CoV-2 spike glycoprotein. Cell 181, 281-292.e6 (2020).

43. Widjaja, I. et al. Towards a solution to MERS: protective human monoclonal antibodies targeting different domains and functions of the MERS-coronavirus spike glycoprotein. Emerg. Microb. Infect. 8, 516-530 (2019).

44. Schmidt, F. et al. Measuring SARS-CoV-2 neutralizing antibody activity using pseudotyped and chimeric viruses. J. Exp. Med. 217, e20201181 (2020).

45. Lang, S. et al. Antibody $27 \mathrm{~F} 3$ broadly targets influenza A group 1 and 2 hemagglutinins through a further variation in VH1-69 antibody orientation on the HA stem. Cell Rep. 20, 2935-2943 (2017).

46. Chen, F., Tzarum, N., Wilson, I. A. \& Law, M. VH1-69 antiviral broadly neutralizing antibodies: genetics, structures, and relevance to rational vaccine design. Curr. Opin. Virol. 34, 149-159 (2019).

47. Avnir, Y. et al. Molecular signatures of hemagglutinin stem-directed heterosubtypic human neutralizing antibodies against influenza A viruses. PLoS Pathog. 10, e1004103 (2014).

48. Gorny, M. K. et al. Preferential use of the VH5-51 gene segment by the human immune response to code for antibodies against the V3 domain of HIV-1. Mol. Immunol. 46, 917-926 (2009).

49. Williams, W. B. et al. HIV-1 VACCINES. Diversion of HIV-1 vaccineinduced immunity by gp41-microbiota cross-reactive antibodies. Science $\mathbf{3 4 9}$, aab1253 (2015)

50. Joyce, M. G. et al. Vaccine-induced antibodies that neutralize group 1 and group 2 influenza A viruses. Cell 166, 609-623 (2016).

51. Walls, A. C. et al. Glycan shield and epitope masking of a coronavirus spike protein observed by cryo-electron microscopy. Nat. Struct. Mol. Biol. 23, 899 (2016).

52. Walls, A. C. et al. Tectonic conformational changes of a coronavirus spike glycoprotein promote membrane fusion. Proc. Natl Acad. Sci. USA 114 11157-11162 (2017).

53. Li, K. et al. Middle East respiratory syndrome coronavirus causes multiple organ damage and lethal disease in mice transgenic for human dipeptidyl peptidase 4. J. Infect. Dis. 213, 712-722 (2016).

54. Ekiert, D. C. et al. Cross-neutralization of influenza A viruses mediated by a single antibody loop. Nature 489, 526-532 (2012).

55. Schmidt, A. G. et al. Viral receptor-binding site antibodies with diverse germline origins. Cell 161, 1026-1034 (2015).
56. Routledge, E., Stauber, R., Pfleiderer, M. \& Siddell, S. G. Analysis of murine coronavirus surface glycoprotein functions by using monoclonal antibodies. J. Virol. 65, 254-262 (1991).

57. Elshabrawy, H. A., Coughlin, M. M., Baker, S. C. \& Prabhakar, B. S. Human monoclonal antibodies against highly conserved HR1 and HR2 domains of the SARS-CoV spike protein are more broadly neutralizing. PLoS ONE 7, e50366 (2012).

58. Lip, K. M. et al. Monoclonal antibodies targeting the HR2 domain and the region immediately upstream of the HR2 of the $S$ protein neutralize in vitro infection of severe acute respiratory syndrome coronavirus. J. Virol. 80, 941-950 (2006).

59. Supekar, V. M. et al. Structure of a proteolytically resistant core from the severe acute respiratory syndrome coronavirus S2 fusion protein. Proc. Natl Acad. Sci. USA 101, 17958-17963 (2004).

60. $\mathrm{Xu}, \mathrm{Y}$. et al. Structural basis for coronavirus-mediated membrane fusion. Crystal structure of mouse hepatitis virus spike protein fusion core. J. Biol. Chem. 279, 30514-30522 (2004).

61. King, L. B. et al. Cross-reactive neutralizing human survivor monoclonal antibody BDBV223 targets the ebolavirus stalk. Nat. Commun. 10, 1-8 (2019).

62. Berger, C. et al. Antigen recognition by conformational selection. FEBS Lett. 450, 149-153 (1999).

63. Rappazzo, C. G. et al. An engineered antibody with broad protective efficacy in murine models of SARS and COVID-19. Preprint at https://doi.org/ 10.1101/2020.11.17.385500 (2020).

64. Song, G. et al. Cross-reactive serum and memory B cell responses to spike protein in SARS-CoV-2 and endemic coronavirus infection. Preprint at https://www.ncbi.nlm.nih.gov/pmc/articles/PMC7523096/(2020).

65. $\mathrm{Li}, \mathrm{Y}$. et al. Linear epitopes of SARS-CoV-2 spike protein elicit neutralizing antibodies in COVID-19 patients. Cell Mol. Immunol. 17, 1095-1097 (2020)

66. Ladner, J. T. et al. Epitope-resolved profiling of the SARS-CoV-2 antibody response identifies cross-reactivity with endemic human coronaviruses. Cell Rep. Med. 2, 100189 (2021).

67. $\mathrm{Xu}, \mathrm{K}$. et al. Epitope-based vaccine design yields fusion peptide-directed antibodies that neutralize diverse strains of HIV-1. Nat. Med. 24, 857-867 (2018).

68. Nachbagauer, R. \& Krammer, F. Universal influenza virus vaccines and therapeutic antibodies. Clin. Microbiol. Infec. 23, 222-228 (2017).

69. Crowe, J. E. Jr Principles of broad and potent antiviral human antibodies: insights for vaccine design. Cell Host Micro. 22, 193-206 (2017).

70. Daramola, O. et al. A high-yielding $\mathrm{CHO}$ transient system: coexpression of genes encoding EBNA-1 and GS enhances transient protein expression. Biotechnol. Prog. 30, 132-141 (2014).

71. Gahn, T. A. \& Sugden, B. An EBNA-1-dependent enhancer acts from a distance of 10 kilobase pairs to increase expression of the Epstein-Barr virus LMP gene. J. Virol. 69, 2633-2636 (1995).

72. Liu, H. F., Ma, J., Winter, C. \& Bayer, R. Recovery and Purification Process Development for Monoclonal Antibody Production (MAbs Ser. 2, Taylor \& Francis, 2010).

73. Hulswit, R. J. G. et al. Human coronaviruses OC43 and HKU1 bind to 9-Oacetylated sialic acids via a conserved receptor-binding site in spike protein domain A. Proc. Natl Acad. Sci. USA 116, 2681-2690 (2019).

74. Raj, V. S. et al. Chimeric camel/human heavy-chain antibodies protect against MERS-CoV infection. Sci. Adv. 4, eaas9667 (2018).

75. Okba, N. M. et al. Severe acute respiratory syndrome coronavirus $2-$ specific antibody responses in coronavirus disease patients. Emerg. Infect. Dis. 26, $1478-1488(2020)$

\section{Acknowledgements}

We thank dr. Yoshiharu Matsuura (Osaka University, Japan) for providing the luciferaseencoding VSV-G pseudotyped VSV $\Delta$ G-luc virus, Volker Thiel (University of Bern, Switzerland) for providing the HCoV-HKU1 spike protein encoding plasmid and Alejandra Tortorici (Institute Pasteur, France) for providing MERS-CoV S ectodomain. We thank Jaap Willem Back from PEPSCAN Presto BV, Lelystad, the Netherlands for his assistance with the spike protein peptide microarray analysis. We thank Ludo Broos for technical support. This study was done within the framework of the Utrecht Molecular Immunology Hub - Utrecht University. Funding: The project was co-financed by a grant from the Zoonotic Anticipation and Preparedness Initiative [ZAPI project; Innovative Medicines Initiative (IMI) grant agreement no. 115760], with the assistance and financial support of IMI and the European Commission, and in-kind contributions from European Federation of Pharmaceutical Industries and Associations partners. This manuscript was part of the research programme of the Netherlands Centre for One Health (www.ncoh.nl). The collaboration project is co-funded by the PPP Allowance made available by Health Holland, Top Sector Life Sciences \& Health, to stimulate publicprivate partnerships. This study was also partially financed by grants from the Ministry of Science and Innovation of Spain (BIO2016-75549-R AEI/FEDER, UE) and NIH (2PO1AIO6O699). The mice used to generate the mAbs produced in this study were provided by Harbour Antibodies BV, a daughter company of Harbour Biomed 
(http://www.harbourbiomed.com). Chunyan Wang was supported by a grant from the China Scholarship Council.

\section{Author contributions}

B.J.B. conceived and coordinated the study. C.W. D.D. and B.J.B. designed the experiments. C.W., R.H., J.G.A., W.L., N.M.A.O., I.A., I.W., B.D., R.F.D., I.S. and D.D. conducted the experiments. D.L.H., O.D., F.G., F.J.M.K., B.L.H., L.E., D.D. and B.J.B. supervised part of the experiments. All authors contributed to the interpretations and conclusions presented. C.W. and B.J.B. wrote the manuscript with comments from all coauthors. All authors participated in editing the manuscript.

\section{Competing interests}

C.W., R.v.H., W.L., I.W., B.v.D., N.M.A.O., F.G., F.J.M.v.K., B.L.H., D.D., and B.J.B. are inventors on a patent application on monoclonal antibodies targeting MERS-CoV (patent publication no.: WO/2020/169755). F.G., D.D. and R.H. are non-substantial interest shareholders in Harbour Biomed and were part of the team that generated the mice. The other authors declare no competing interests.

\section{Additional information}

Supplementary information The online version contains supplementary material available at https://doi.org/10.1038/s41467-021-21968-w.

Correspondence and requests for materials should be addressed to B.-J.B.

Peer review information Nature Communications thanks Dimiter Dimitrov and the other, anonymous, reviewer(s) for their contribution to the peer review of this work.

Reprints and permission information is available at http://www.nature.com/reprints

Publisher's note Springer Nature remains neutral with regard to jurisdictional claims in published maps and institutional affiliations.

\section{(c) (i)} adaptation, distri Commons license, and indicate if changes were made. The images or other third party material in this article are included in the article's Creative Commons license, unless indicated otherwise in a credit line to the material. If material is not included in the article's Creative Commons license and your intended use is not permitted by statutory regulation or exceeds the permitted use, you will need to obtain permission directly from the copyright holder. To view a copy of this license, visit http://creativecommons.org/ licenses/by/4.0/.

(C) The Author(s) 2021 